\title{
IDEALS ASSOCIATED TO DEFORMATIONS OF SINGULAR PLANE CURVES
}

\author{
STEVEN DIAZ AND JOE HARRIS
}

\begin{abstract}
We consider in this paper the geometry of certain loci in deformation spaces of plane curve singularities. These loci are the equisingular locus $E S$ which parametrizes equisingular or topologically trivial deformations, the equigeneric locus $E G$ which parametrizes deformations of constant geometric genus, and the equiclassical locus $E C$ which parametrizes deformations of constant geometric genus and class. (The class of a reduced plane curve is the degree of its dual.)

It was previously known that the tangent space to ES corresponds to an ideal called the equisingular ideal and that the support of the tangent cone to $E G$ corresponds to the conductor ideal. We show that the support of the tangent cone to $E C$ corresponds to an ideal which we call the equiclassical ideal. By studying these ideals we are able to obtain information about the geometry and dimensions of $E S, E C$, and $E G$. This allows us to prove some theorems about the dimensions of families of plane curves with certain specified singularities.
\end{abstract}

1. Introduction. We consider in this paper the geometry of certain loci in deformation spaces of plane curve singularities. Specificially, if $p$ is a singular point of a reduced plane curve $D$, then we have an étale versal deformation of $(D, p)$ (defined precisely in $\S 3$, see $[\mathbf{A 1}]$ ).

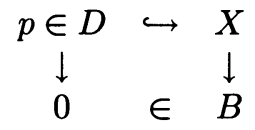

In the deformation space $B$ we introduce three loci:

(1) the equisingular locus $E S \subset B$ which parametrizes equisingular deformations (the condition of equisingularity may be thought of as "topologically trivial"; a precise definition is given in $\S 3$ );

(2) the equigeneric or $\delta$-constant locus $E G \subset B$ which parametrizes deformations of $(D, p)$ of constant geometric genus;

(3) the equiclassical locus $E C \subset B$ which parametrizes deformations of $(D, p)$ of constant geometric genus and class (the class of a reduced plane curve is the degree of its dual).

For example, if $(D, p)$ is an ordinary cusp with equation $D=\left\{y^{2}-x^{3}=0\right\}$, $p=(0,0)$, then we may take for $B$ the affine plane with coordinates $a$ and $b$, and

Received by the editors April 13, 1987 and, in revised form, July 22, 1987.

1980 Mathematics Subject Classification (1985 Revision). Primary 14B07; Secondary 14H10, $14 \mathrm{H} 20$.

The first author was almost totally supported by a National Science Foundation Mathematical Sciences Postdoctoral Research Fellowship. The second author was partially supported by National Science Foundation grant number DMS-84-02209. 
for $X$ the deformation

$$
X=\left\{y^{2}-x^{3}-a x-b=0\right\} \subset \mathbf{A}_{x, y}^{2} \times \mathbf{A}_{a, b}^{2} .
$$

The equisingular locus $E S$ and the equiclassical locus $E C$ are both equal to the origin $(0,0) \in B$, and the equigeneric locus $E G$ is the discriminant curve $\left\{4 a^{3}+27 b^{2}=0\right\} \subset B$.

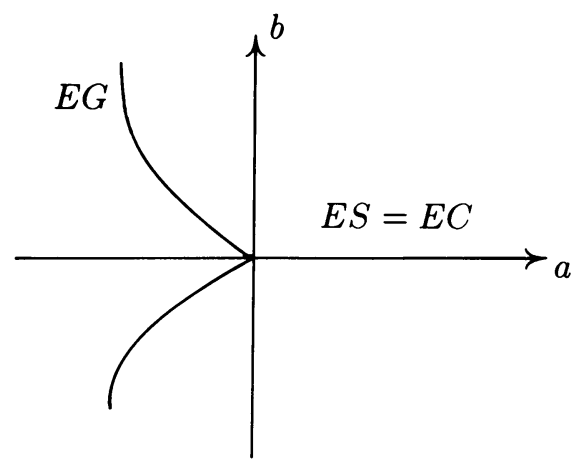

The simplest example in which $E S$ is positive dimensional is that of an ordinary four fold point, say $D=\left\{x^{4}+y^{4}=0\right\}, p=(0,0)$. Here $B$ may be taken to be a 9 -plane with coordinates $a, b, \ldots, i$ and $X$ the deformation

$$
\begin{aligned}
X & =\left\{a+b x+c y+d x^{2}+e x y+f y^{2}+g x^{2} y+h x y^{2}+i x^{2} y^{2}+x^{4}+y^{4}=0\right\} \\
& \subset \mathbf{A}_{x, y}^{2} \times \mathbf{A}_{a, \ldots, i}^{9} .
\end{aligned}
$$

In this case $E S$ is the coordinate axis $a=\cdots=h=0$ which corresponds to motions of the four lines of $D$ around the origin, $p$, changing the cross-ratio of their slopes. $E G$ and $E C$ on the other hand are both equal to the more complicated locus corresponding to motions of the four lines of $D$ not necessarily around the origin. Observe that while the equations of $E G=E C$ are messy, we can see without difficulty that, in a neighborhood of $(0, \ldots, 0) \in B, E G=E C$ is three dimensional and smooth with tangent space $a=\cdots=f=0$.

A case where these three loci are all different from each other is a ramphoid cusp, say $D=\left\{y^{2}+x^{5}=0\right\}, p=(0,0)$. Here $B$ may be taken to be a 4-plane with coordinates $a, b, c, d$ and $X$ the deformation

$$
X=\left\{y^{2}+x^{5}+a x^{3}+b x^{2}+c x+d=0\right\} \subset \mathbf{A}_{x, y}^{2} \times \mathbf{A}_{a, \ldots, d}^{6} .
$$

With fairly straightforward calculations one may show the following. $E S$ is the origin. $E C$ is one dimensional with parametric equations $a=-\frac{15}{4} t^{2}, b=-\frac{5}{4} t^{3}$, $c=\frac{15}{4} t^{4}, d=\frac{9}{4} t^{5}$. A general point of $E C$ corresponds to a curve with one ordinary node and one ordinary cusp. $E G$ is two dimensional with parametric equations $a=-3 s^{2}-4 s t-3 t^{2}, b=-2 s^{3}-8 s^{2} t-8 s t^{2}-2 t^{3}, c=-4 s^{3} t-7 s^{2} t^{2}-4 s t^{3}$, $d=-2 s^{3} t^{2}-2 s^{2} t^{3}$. A general point of $E G$ corresponds to a curve with two ordinary nodes.

Here is a brief summary of what is known about the spaces $B, E S, E C$, and $E G$. 
(i) $B$ is smooth, with tangent space $T_{0} B$ at 0 naturally identified with the quotient $\mathscr{O}_{D, p} / J$ where $\mathscr{O}_{D, p}$ is the local ring of $D$ at $p$ and $J \subset \mathscr{O}_{D, p}$ is the Jacobian ideal. (If $D$ is given locally by $D=\{f=0\}$, then $J$ is the ideal generated by the partial derivatives $\partial f / \partial x, \partial f / \partial y$.) See $[\mathbf{A 2}, \mathbf{S 1}, \mathbf{S 2}]$.

(ii) $E S$ is also smooth at 0 , with tangent space corresponding via the identification $T_{0} B=\mathscr{O}_{D, p} / J$ to an ideal $I \subset \mathscr{O}_{D, p}$ called the equisingular ideal. See [T2, W].

(iii) $E G$ may be singular at 0 (as the example we gave for the cusp shows) though it is always locally irreducible at 0 . (This follows from results of $[\mathbf{A C}]$ and will be explained in §4.) The support of the tangent cone to $E G$ at 0 is always a linear space. Specifically, in terms of the identification $T_{0} B=\mathscr{O}_{D, p} / J$, we have that the support of the tangent cone to $E G$ at 0 is identified with $A / J$, where $A \subset \mathscr{O}_{D, p}$ is the conductor ideal of $\mathscr{O}_{D, p}$. (That is, $A$ is the annihilator of the $\mathscr{O}_{D, p}$-module $\widetilde{\mathscr{O}}_{D, p} / \mathscr{O}_{D, p}$ where $\widetilde{\mathscr{O}}_{D, p}$ is the integral closure of $\mathscr{O}_{D, p}$ in its total quotient ring.) See [T1]. We give essentially the same proof as Tessier in $\S 4$. In particular we note that

$$
\operatorname{codim}(E G \subset B)=\operatorname{length}\left(\widetilde{\mathscr{O}}_{D, p} / \mathscr{O}_{D, p}\right)=\delta
$$

where $\delta$ is the number of adjoint conditions imposed by the singularity.

(iv) $E C$ may be singular at 0, as the example we gave for a ramphoid cusp shows. The support of the tangent cone to $E C$ at 0 is always a linear space. Specifically, in terms of the identification $T_{0} B=\mathscr{O}_{D, p} / J$, we have that the support of the tangent cone to $E C$ at 0 is identified with $H / J$, where $H \subset \mathscr{O}_{D, p}$ is an ideal which we call the equiclassical ideal. $H$ may be described as follows. Let $\mathscr{O}_{D, p}, \widetilde{\mathscr{O}}_{D, p}$, and $J$ be as in (i) and (iii). Then $H$ is the contraction to $\mathscr{O}_{D, p}$ of the extension to $\widetilde{\mathscr{O}}_{D, p}$ of $J$. $E C=E G$ if and only if all the analytic branches of the singularity of $D$ at $p$ are nonsingular. These results will be proven in $\S 5$.

We see from this that we have a sequence of four ideals in $\mathscr{O}_{D, p}, J \subset I \subset H \subset$ $A \subset \mathscr{O}_{D, p}$, that reflect the geometry of deformations of $(D, p) .(J, H$, and $A$ of course have other simpler definitions while $I$ is defined solely by its relation to deformations.)

We also show that the only singularities for which $E C$ is equal to $E S$ are the ordinary node and cusp. This allows us to obtain the following two results.

(1.1) THEOREM. Any reduced plane curve singularity may be deformed to ordinary nodes and cusps in a flat family in which the genus and class of the fibers remain constant.

(1.2) THEOREM. Let $\mathbf{P}^{N}$ be the projective space that parametrizes curves in $\mathbf{P}^{2}$ of degree d. Let $Z \subset \mathbf{P}^{N}$ be the locally closed subset of reduced and irreducible curves of geometric genus $g$ and class $c$. Assume that $c \geq 2 g-1$. Let $D$ be a general element of any component of $Z$. Then any singularity of $D$ is either an ordinary node or an ordinary cusp.

These two theorems resulted from questions posed to the authors by William Fulton, to whom they are grateful.

In the last section of this paper, we obtain some estimates on the relative size of these ideals (e.g. $\operatorname{dim}_{\mathbf{C}}(A / I) \geq b-2$, where $b$ is the number of analytic branches of $D$ at $p$ ). In particular we classify all singularities for which $\operatorname{dim}_{\mathbf{C}}(A / I)$ (equivalently, 
the codimension of $E S$ in $E G$ ) is one or two. This in turn yields statements about singularities occurring in families of plane curves, which was the original motivation for this work. Specifically, let $\mathbf{P}^{N}$ be the space of all plane curves of degree $d$, and $V \subset \mathbf{P}^{N}$ the locally closed subset of reduced and irreducible curves of geometric genus $g$. We already know

(1.3) THEOREM [AC, $\mathbf{Z 1}, \mathbf{H}] . V$ is irreducible and the general member $D \in V$ is a curve with exactly $n=\frac{1}{2}(d-1)(d-2)-g$ nodes as its only singularities.

We would like to say something about the codimension in which curves with various other singularities occur. The final result of this paper is the following theorem.

(1.4) THEOREM. If $W \subset V$ is any subvariety of codimension 1 , and $D \in W$ a general point, then the singularities of $D$ are either

(i) $n$ nodes,

(ii) $n-1$ nodes and one cusp $\left(y^{2}-x^{3}\right)$,

(iii) $n-2$ nodes and one tacnode $\left(y\left(y-x^{2}\right)\right)$, or

(iv) $n-3$ nodes and one ordinary triple point $\left(x^{3}-y^{3}\right)$.

Throughout this paper the ground field is the field of complex numbers.

The authors would like to thank the following people for helpful discussions during the investigations which led to this paper: Michael Artin, David Eisenbud, Gert-Martin Greuel, and Rennie Mirollo.

2. Geometric genus in flat families. For a reduced projective curve $D$ we define the geometric genus of $D$ to be the arithmetic genus of the normalization of D.

(2.1) In what follows we let

$$
\begin{aligned}
& X \subset \mathbf{P}^{m} \times Y \\
& \downarrow \pi \\
& Y
\end{aligned}
$$

be a flat family of projective curves with all fibers reduced. Also assume that $X$ and $Y$ are reduced separated schemes of finite type over $\mathbf{C}$. Define the function $\phi_{\pi}$ on $Y$ by letting $\phi_{\pi}(y)$ be the geometric genus of the fiber of $\pi$ over $y \in Y$.

(2.2) Lemma. Assume that $Y$ is a nonsingular curve. Let $f: X^{\prime} \rightarrow X$ be the normalization map. Then $\pi \circ f: X^{\prime} \rightarrow Y$ is a flat family of projective curves with all fibers reduced.

ProOF. For flatness, [Ha], Proposition III.9.7] says that every component of $X$ dominates $Y$. This says that every component of $X^{\prime}$ dominates $Y$. Applying [Ha, Proposition III.9.7] again we conclude that $\pi \circ f$ is flat.

$X$ must be nonsingular at any nonsingular point of any fiber of $\pi$. Therefore a fiber of $\pi \circ f$ cannot have any multiple components. We must eliminate the possibility of isolated nonreduced points. Each component of $X^{\prime}$ is an irreducible normal variety and it is sufficient to check reducedness on each component of $X^{\prime}$. The base of the family is a nonsingular curve, so we may assume that the ideal of the fiber is principal. Since in a normal Noetherian domain principal ideals are unmixed we are done. 
(2.3) COROLLARY. Under the assumptions of $(2.2), \phi_{\pi}$ is lower semicontinuous in the Zariski topology.

ProOF. We have two functions on $Y, \phi_{\pi}$ and $\phi_{\pi \circ f}$. From (2.2) we see that all fibers of $\pi \circ f$ are either normalizations or partial normalizations of the corresponding fibers of $\pi$. We conclude that $\phi_{\pi \circ f}=\phi_{\pi}$. Since $X^{\prime}$ is normal it has at most finitely many singular points. Applying the theorem of generic smoothness we conclude that all but finitely many fibers of $\pi \circ f$ are nonsingular. Since flatness implies that the arithmetic genus of the fibers remains constant we conclude that $\phi_{\pi \circ f}$ is constant on the Zariski open set where all fibers are nonsingular. Finally, for a reduced singular curve the geometric genus is strictly less than the arithmetic genus so $\phi_{\pi \circ f}$ decreases at singular fibers.

(2.4) PROPOSITION. Under the general assumptions in (2.1) $\phi_{\pi}$ is lower semicontinuous in the Zariski topology.

Proof. Claim 1. There exists a dense Zariski open subset $U \subset Y$ on which $\phi_{\pi}$ is equal to a constant $g$.

PROOF OF CLAIM. Let $U_{1}=$ the nonsingular points of $Y$. Let $V$ be the normalization of $\pi^{-1}\left(U_{1}\right)$ and $f: V \rightarrow U_{1}$ the induced family of curves. Since $V$ is normal its singularities form a closed set $A$ of codimension at least 2 in $V$. Since $f$ is proper $U_{1}-f(A)$ is a dense Zariski open set of $U_{1}$. By generic smoothness we obtain a dense Zariski open $U \subset U_{1}-f(A)$ on which the morphism $f: f^{-1}(U) \rightarrow U$ is smooth. The fibers of $f$ are just the normalizations of the corresponding fibers of $\pi$. Thus $\phi_{\pi}$ is constant on $U$.

Claim 2. If $y \in Y-U$ then $\phi_{\pi}(y) \leq g$.

PROOF OF CLAIM. Let $Z$ be a curve on $Y$ through $y$ whose generic point lies in $U$. Let $Z^{\prime}$ be the normalization of $Z$. Via base change the family over $Y$ gives a family over $Z^{\prime}$. Corollary (2.3) applied to the family over $Z^{\prime}$ proves the claim.

For $n \in \mathbf{Z}$ set $B(n)=\left\{y \in Y: \phi_{\pi}(y) \leq n\right\}$. We wish to show that $B(n)$ is Zariski closed. Fix $n$. If $B(n)=Y$ we are done. If not applying Claims 1 and 2 we obtain a Zariski closed set $Y_{1} \varsubsetneqq Y,\left(Y_{1}=Y-U\right)$ such that $B(n) \subset Y_{1}$. If $B(n)=Y_{1}$ we are done. If not apply the claims to the family $\pi: \pi^{-1}\left(Y_{1}\right) \rightarrow Y_{1}$ and obtain a Zariski closed $Y_{2} \varsubsetneqq Y_{1}$ with $B(n) \subset Y_{2}$. Since $Y$ is assumed to be of finite type over $\mathrm{C}$ and hence Noetherian this process must terminate, so eventually $B(n)=Y_{k}$.

(2.5) THEOREM [T1, p. 80]. Under the general assumptions of (2.1) assume further that $Y$ is normal and $\phi_{\pi}$ is constant. Let $f: X^{\prime} \rightarrow X$ be the normalization map. Then $\pi \circ f: X^{\prime} \rightarrow Y$ is a smooth family of curves. Each fiber of $\pi \circ f$ is the normalization of the corresponding fiber of $\pi$.

(2.6) EXAMPLE. This example shows that if the base of a flat family of reduced curves is not normal, then even if the geometric genus of the fibers is constant there may fail to exist a smooth family of curves over the same base that simultaneously normalizes all the fibers of the original family.

Consider the family

$$
\begin{aligned}
\text { Spec } C & {\left[t^{2}, t^{3}\right] \times \mathbf{P}_{x, y, z}^{2} \supset \mathscr{C}=\left\{y^{2} z-x^{3}-3 t^{2} x z^{2}-2 t^{3} z^{3}=0\right\} } \\
& \downarrow \pi \\
\text { Spec } \mathbf{C}\left[t^{2}, t^{3}\right] &
\end{aligned}
$$


It is easy to check that the geometric genus is constant, equal to zero, in this family. Let us focus our attention near the nonnormal point of Spec $\mathbf{C}\left[t^{2}, t^{3}\right]$ and near the singular point of the fiber over that point. We do this by taking completions.

$$
\begin{gathered}
\text { Spec } \mathbf{C}\left[\left[t^{2}, t^{3}\right]\right] \times \operatorname{Spec} \mathbf{C}[[x, y]] \supset \mathscr{C}^{\prime}=\left\{y^{2}-x^{3}-3 t^{2} x-2 t^{3}=0\right\} \\
\downarrow \\
\quad \operatorname{Spec} \mathbf{C}\left[\left[t^{2}, t^{3}\right]\right]
\end{gathered}
$$

We wish to show that there does not exist a commutative diagram

$$
\begin{gathered}
\text { Spec } \mathbf{C}[[s]] \times \operatorname{Spec} \mathbf{C}\left[\left[t^{2}, t^{3}\right]\right] \rightarrow \mathscr{C}^{\prime} \\
\downarrow \\
\operatorname{Spec} \mathbf{C}\left[\left[t^{2}, t^{3}\right]\right]
\end{gathered}
$$

simultaneously normalizing all the fibers of $\pi$. The existence of such a family implies the existence of power series

$$
\begin{aligned}
& X=a_{0}\left(t^{2}, t^{3}\right)+a_{1}\left(t^{2}, t^{3}\right) s+a_{2}\left(t^{2}, t^{3}\right) s^{2}+\cdots, \\
& Y=b_{0}\left(t^{2}, t^{3}\right)+b_{1}\left(t^{2}, t^{3}\right) s+b_{2}\left(t^{2}, t^{3}\right) s^{2}+\cdots
\end{aligned}
$$

such that

$$
Y^{2}-X^{3}-3 t^{2} X-2 t^{3}=0 .
$$

By change of coordinates on $\mathbf{C}[[s]]$ we may assume that when $t=0, X=s^{2}$, $Y=s^{3}$. This means that $a_{0}$ and $b_{0}$ have no constant term. Looking at the $s^{0}$ term in (2.7) we get $b_{0}^{2}-a_{0}^{3}-3 t^{2} a_{0}-2 t^{3}=0$. This is impossible because $b_{0}^{2}, a_{0}^{3}$, and $3 t^{2} a_{0}$ have no $t^{3}$ terms.

Suppose one has a family as in (2.1) in which $\phi_{\pi}$ is constant, equal to $g$, and all fibers are irreducible. Under such circumstances one will sometimes hear someone say, "Consider the natural morphism from $Y$ to $\mathscr{M}_{g}$, the moduli space of curves of genus $g$." In view of (2.6) one may see that this morphism does not exist in general. Theorem (2.5) says that there is a natural morphism from the normalization of $Y$ to $\mathscr{M}_{g}$.

(2.8) This example was suggested by Bernard Teissier. In it we show that in a family such as (2.1) when the geometric genus is not constant and $Y$ has dimension greater than one, normalizing $X$ may fail to yield a flat family of reduced curves even when $Y$ is nonsingular. We work locally. Let $X \subset \mathbf{C}^{N}, N \geq 5$, be a normal variety of dimension 3 which is not Cohen-Macaulay at the origin. Let $f: X \rightarrow \mathbf{C}^{4}$ be a generic projection, $h: f(X) \rightarrow \mathbf{C}^{2}$ another generic projection, and $g$ the composition of $f$ and $h$. Assume origins always map to origins. Because $f(X)$ is a hypersurface it is easy to check that, for sufficiently general choices of $f$ and $h, h: f(X) \rightarrow \mathbf{C}^{2}$ will be a flat family of reduced plane curves near the origin and the map $f$ is the normalization map. The family $g: X \rightarrow \mathbf{C}^{2}$ cannot be a flat family of reduced curves. Flatness would say the regular sequence defining the origin in $\mathbf{C}^{2}$ pulls back to give a regular sequence defining $g^{-1}(0,0)$ in $X$. If $g^{-1}(0,0)$ was reduced then this regular sequence of length two would extend to a regular sequence of length three everywhere along $g^{-1}(0,0)$ contradicting the fact that $X$ is not Cohen-Macaulay at the origin.

3. Deformation spaces. Let $D=\{f(x, y)=0\}$ be a reduced curve in $\mathbf{A}^{2}$. Assume that $p=(0,0)$ is a point of $D$. Let $J$ be the Jacobian ideal of $f$, that is the 
ideal in $\mathbf{C}[x, y]$ generated by $f, \partial f / \partial x$, and $\partial f / \partial y$. Choose $g_{1}(x, y), \ldots, g_{m}(x, y) \in$ $\mathbf{C}[x, y]$ so that their images in $\mathbf{C}[x, y] / J$ form a basis for this vector space. Consider the following family of curves

$$
\begin{gathered}
\operatorname{Spec} \mathbf{C}[x, y] \times \operatorname{Spec} \mathbf{C}\left[t_{1}, \ldots, t_{m}\right] \supset \mathscr{C}=:\left\{f+\sum t_{i} g_{i}=0\right\} \\
\downarrow \text { projection } \swarrow \pi \\
\operatorname{Spec} \mathbf{C}\left[t_{1}, \ldots, t_{m}\right]=: B
\end{gathered}
$$

From [A1, p. 165; S1 and S2] one may conclude that this family satisfies (3.2) through (3.6).

(3.2) VERSALITY. Given the following data: a flat family of reduced curves $f: X \rightarrow Y$ where $X$ and $Y$ are of finite type over $\mathbf{C}$, a closed point $y \in Y$, a finite number of closed points $x_{1}, \ldots, x_{n} \in f^{-1}(y)$, and an isomorphism of an étale neighborhood of $\left\{x_{1}, \ldots, x_{n}\right\}$ in $f^{-1}(y)$ with an étale neighborhood of a finite set of points $p_{1}, \ldots, p_{n}$ in $D$, then there exist étale neighborhoods $V$ of $(0, \ldots, 0)$ in $B$, $V^{\prime}$ of $y$ in $Y, W$ of $\left\{p_{1}, \ldots, p_{n}\right\}$ in $V \times_{B} \mathscr{C}$, and $W^{\prime}$ of $\left\{x_{1}, \ldots, x_{n}\right\}$ in $V^{\prime} \times_{Y} X$, a morphism $g: V^{\prime} \rightarrow V$, and an isomorphism $\phi: W^{\prime} \rightarrow W \times_{V} V^{\prime}$ such that diagram (3.3) commutes.

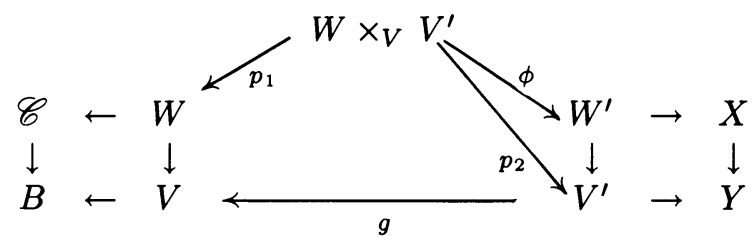

(3.4) Miniversality. If $p: \mathscr{E} \rightarrow \mathscr{F}$ is any other family of curves which is an étale versal deformation for $D$ (that is $p: \mathscr{E} \rightarrow \mathscr{F}$ can take the place of $\pi: \mathscr{C} \rightarrow B$ in (3.2)) then the dimension of $\mathscr{F}$ is greater than or equal to the dimension of $B$.

(3.5) OPENNESS OF VERSALITY. Furthermore, there exists a Zariski open subset $U$ of $B$ containing $(0, \ldots, 0)$ such that $\pi: \mathscr{C} \rightarrow B$ is an étale versal deformation space for all fibers over closed points of $U$.

(3.6) Let $y$ be a closed point of the set $U$ of (3.5) and let $x_{1}, \ldots, x_{n} \in \pi^{-1}(y)$ be a finite set of singular points of $\pi^{-1}(y)$. Suppose we can find reduced curves $D_{1}, \ldots, D_{n}$ in $\mathbf{A}^{2}$ such that $(0,0)$ is the unique singular point of each $D_{i}$ and an étale neighborhood of $(0,0)$ in $D_{i}$ is isomorphic to an étale neighborhood of $x_{i}$ in $\pi^{-1}(y)$. (Later in this section we shall see that we can always do this.) Let $\pi_{i}: \mathscr{C}_{i} \rightarrow B_{i}$ be the étale versal deformation for $D_{i}$ constructed as in (3.1). Using (3.2) and (3.5) we get étale neighborhoods $V_{i}^{\prime}$ of $(0, \ldots, 0)$ in $B$ and $V_{i}$ of $(0, \ldots, 0)$ in $B_{i}$ and morphisms $g_{i}: V_{i}^{\prime} \rightarrow V_{i}$ making a diagram like (3.3) commute. This gives a morphism $g$ from the intersection of the $V_{i}^{\prime}$ 's to the product of the $V_{i}$ 's. The morphism $g$ (after possibly shrinking the étale neighborhoods) is surjective.

When we speak of the étale versal deformation space of a reduced plane curve we mean the family of (3.1). When we speak of an étale versal (or miniversal) deformation space of a reduced plane curve we mean any flat family satisfying (3.2) (or (3.2) and (3.4)).

Many authors have preferred to work with the formal versal deformation of the singularity of $D$ at $p$ rather than the étale versal deformation of $D$. To construct 
the formal versal deformation simply go to (3.1) and replace polynomial rings with power series rings wherever you see them. Call the resulting family of algebroid plane curves $\pi^{\prime}: \mathscr{C}^{\prime} \rightarrow B^{\prime}$. It has the following properties. See $[\mathbf{S 1}, \mathbf{S 2}$, and A2, $\S 4]$.

Let $Y$ be a scheme which is the spectrum of either an Artin local ring, a power series ring (over $\mathbf{C}$ ), or the quotient of a power series ring by an ideal. Let $y$ be the unique closed point of $Y$. Given any flat family $f: X \rightarrow Y$ of reduced algebroid plane curves together with an isomorphism $\zeta: \pi^{\prime-1}(0, \ldots, 0) \rightarrow f^{-1}(y)$, then there exists a morphism $g: Y \rightarrow B^{\prime}$ and an isomorphism $\psi: X \rightarrow Y \times_{B^{\prime}} \mathscr{C}^{\prime}$ making diagram (3.7) commute. It is even possible to write down the map $g$ explicitly, see [KS].

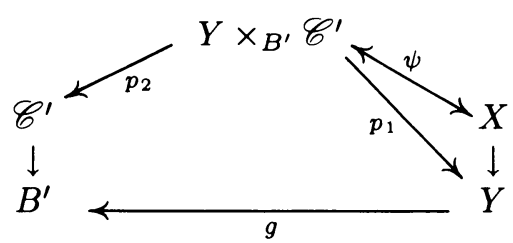

(3.8) If furthermore $Y=\operatorname{Spec} \mathbf{C}[\varepsilon] /\left(\varepsilon^{2}\right)$ then there is a unique $g$ such that $p_{2} \circ \psi \circ \zeta$ is the identity map on $\pi^{\prime-1}(0, \ldots, 0)$. This says that $\pi^{\prime}: \mathscr{C}^{\prime} \rightarrow B^{\prime}$ is a universal first order deformation.

Clearly these two deformation spaces are closely related. In this section we will study certain subschemes of $B$ and $B^{\prime}$ that parametrize deformations that preserve certain properties of the singularity of $D$ at $p$. To get started we must recall several definitions.

Let $D$ containing the point $p$ and $E$ containing the point $q$ be two reduced curves on a smooth surface $S$.

(3.9) DEFinition. We say that the singularity of $D$ at $p$ is analytically isomorphic to the singularity of $E$ at $q$ if the complete local ring of $D$ at $p$ is isomorphic to the complete local ring of $E$ at $q$.

Zariski [Z2, p. 508] defines an algebroid plane curve to be a local ring of the form $\mathbf{C}[[x, y]] /(f)$, where $f=f(x, y)$ is a nonunit element of $\mathbf{C}[[x, y]]$ free from multiple factors. The branches of this curve are just the algebroid plane curves corresponding to irreducible factors of $f$. It is sometimes more convenient to think of Spec $\mathbf{C}[[x, y]] /(f)$ as the algebroid plane curve.

Returning to the curves $D$ and $E$ on a smooth surface $S$ note that the complete local ring of $S$ at $p$ is isomorphic to $\mathbf{C}[[x, y]]$. Let $f(x, y)$ be a local equation for $D$ near $p$. Then $\mathbf{C}[[x, y]] /(f)$ is called the algebroid plane curve associated to $D$ at $p$; proceed similarly for $E$ at $q$. Zariski [Z2, pp. 510-514] gives three equivalent definitions for what it means for two plane algebroid curves to have equivalent singularities. We repeat one of them here. We will then say that two singularities of reduced curves on smooth surfaces are equivalent if their associated algebroid plane curves (obtained as just described) have equivalent singularities in the sense of Zariski.

Let $D=\mathbf{C}[[x, y]] /(f)$ with branches $\gamma_{1}, \ldots, \gamma_{h}$ and $E$ with branches $\delta_{1}, \ldots, \delta_{h}$ be two algebroid plane curves. 
(3.10) Definition [Z2, p. 510]. A $(1,1)$ mapping $\pi$ of the set of branches of $D$ onto the set of branches of $E$ is said to be a tangentially stable pairing $\pi: D \rightarrow E$ between the branches of $D$ and those of $E$, if the following condition is satisfied: given any two branches $\gamma_{i}$ and $\gamma_{j}$ of $D$, the corresponding branches $\pi\left(\gamma_{i}\right)$ and $\pi\left(\gamma_{j}\right)$ of $E$ have the same tangent if and only if $\gamma_{i}$ and $\gamma_{j}$ have the same tangent.

If we blow up the origin in Spec $\mathbf{C}[[x, y]]$ the proper transform of Spec $\mathbf{C}[[x, y]] /(f)$ may have several connected components. Each of them can be considered to be an algebroid plane curve; call them $D_{\nu}^{\prime}, \nu=1,2, \ldots$ Similarly one can obtain $E_{\nu}^{\prime}$, $\nu=1,2, \ldots$. Given a tangentially stable pairing $\pi: D \rightarrow E$ one may choose the numbering $\nu$ so that $\pi$ induces pairings $\pi_{\nu}^{\prime}$ of the branches of $D_{\nu}^{\prime}$ and $E_{\nu}^{\prime}$. One may now define equivalence of algebroid plane curves by induction on the number of blowups required to resolve the singularity. When this number is zero we say an equivalence is a pairing of the unique branch of $D$ with the unique branch of $E$. Both branches are of course nonsingular.

(3.11) Definition [Z2, p. 511]. An equivalence $\pi: D \rightarrow E$ is a pairing $\pi$ between the branches of $D$ and the branches of $E$ having the following properties:

(1) $\pi$ is tangentially stable.

(2) If $\delta_{j}=\pi\left(\gamma_{i}\right)(i=1,2, \ldots, h)$, then $\operatorname{mult}_{p}\left(\gamma_{i}\right)=\operatorname{mult}_{q}\left(\delta_{j}\right)$.

(3) The pairing $\pi^{\prime}: D_{\nu}^{\prime} \rightarrow E_{\nu}^{\prime}(\nu=1,2, \ldots)$ is an equivalence.

(3.12) DEFINITION. Let $D$ be a reduced plane algebroid curve and $p$ the closed point of $D$. Let $f: D^{\prime} \rightarrow D$ be the normalization map, $\mathscr{O}$ the local ring of $D$ at $p$, $A$ the conductor ideal of $D$ at $p$ (as defined in the introduction), $k$ the residue field of $D$ at $p(k \cong \mathbf{C})$, and $D_{1}^{\prime}, \ldots, D_{n}^{\prime}$ the irreducible components of $D^{\prime}$. Define

$$
\begin{gathered}
\delta(p)=\operatorname{dim}_{k}(\mathscr{O} / A), \quad m(p)=\sum_{i=1}^{n}\left(\operatorname{mult}_{p}\left(f\left(D_{i}^{\prime}\right)\right)-1\right), \\
\kappa(p)=2 \delta(p)+m(p) .
\end{gathered}
$$

(Notice that $m(p)$ is the degree of the first Fitting ideal of the sheaf of relative differentials of $D^{\prime}$ over $D$.) For any reduced curve $D$ on a smooth surface and $p$ a closed point of $D$ define $\delta(p), m(p)$, and $\kappa(p)$ to be the corresponding numbers for the algebroid plane curve associated to $D$ at $p$. Finally define $\delta(D)$ to be the sum over all closed points $p$ of $D$ of $\delta(p)$, similarly for $m(D)$ and $\kappa(D)$. (These sums are finite because terms of nonsingular points are zero.)

It is an easy-to-prove classical fact that if $D$ is a reduced irreducible curve of degree $d$ in $\mathbf{P}^{2}$ then the geometric genus of $D$ equals $\frac{1}{2}(d-1)(d-2)-\delta(D)$ and the class of $D$, that is the degree of the dual of $D$, equals $d(d-1)-\kappa(D)$.

(3.13) Definition. Consider a flat family of reduced curves in $\mathbf{A}^{2}$.

$$
\begin{aligned}
& X \subset \operatorname{Spec} \mathbf{C}[x, y] \times Y \\
& \downarrow \\
& Y
\end{aligned}
$$

We allow $Y$ to be any separated scheme over $\mathbf{C}$, not necessarily reduced or of finite type.

(1) This family is trivial if given any closed point $y \in Y$ there exists an isomorphism $\phi: X \rightarrow Y \times p^{-1}(y)$ that makes the following diagram commute. 


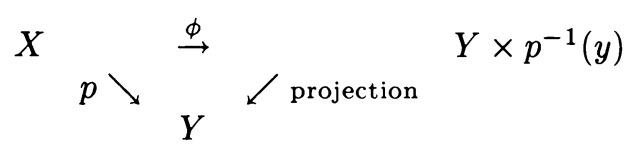

(2) We say the family is equisingular under these conditions.

(a) There exists a finite number of disjoint sections of the family, the union of whose images contains the locus of singular points of fibers, and $X$ is equimultiple (i.e. normally flat) along these sections.

(b) If, in addition, all the singular points of fibers are ordinary double points we say the family is equisingular. If not, blow up the sections.

(c) Now we require that in the family of reduced total transforms there exist sections lying over the former sections (at least one over each former section) satisfying (a). Then return to (b).

(3) We say a family is locally trivial (equisingular) in the Zariski (étale) topology if: (note that this is meant to read as four different definitions) for each closed point $y \in Y$ and each closed point $x \in p^{-1}(y)$ there exist Zariski (étale) open neighborhoods $U$ of $y$ in $Y$ and $V$ of $x$ in $p^{-1}(U)$ such that the induced family $V \rightarrow U$ is trivial (equisingular).

(4) Let $y \in Y$ be a closed point, $\mathscr{O}$ its local ring, and $\mathfrak{m}$ its maximal ideal. For each positive integer $n$ set $Y_{n}=\operatorname{Spec} \mathscr{O} / \mathfrak{m}^{n}$. We then get a family $p_{n}: X \times_{Y} Y_{n} \rightarrow$ $Y_{n}$. We say that $p: X \rightarrow Y$ is formally locally trivial (equisingular) at $y$ if for all $n$ the family $p_{n}$ is trivial (equisingular) in the Zariski topology. We say $p: X \rightarrow Y$ is formally locally trivial (equisingular) if it is at all closed points of $Y$.

(5) Assume that $Y$ is reduced and the locus of singular points of fibers is proper over $Y$. For any point $y \in Y$ closed or not the geometric fiber over $y$ is the curve $\bar{X}_{y}$ obtained by tensoring $f^{-1}(y)$ with the algebraic closure of the residue field of $y$. Define $\delta(y), \kappa(y)$, and $m(y)$ to be respectively $\delta\left(\bar{X}_{y}\right), \kappa\left(\bar{X}_{y}\right)$, and $m\left(\bar{X}_{y}\right)$. We say the family $p: X \rightarrow Y$ is equigeneric if $\delta(y)$ is constant on $Y$ and equiclassical if $\delta(y)$ and $\kappa(y)$ are constant on $Y$.

Notice that one could make these same definitions for a family of algebroid plane curves, or one may replace Spec $\mathbf{C}[x, y]$ with any nonsingular surface.

The definition of equisingular follows that of [W, pp. 143-144]. The definition of formally locally trivial follows that given in [Ta, pp. 113-114].

We now investigate the relationship between formally locally trivial families and families in which all fibers over closed points have analytically isomorphic singularities. Much of this has already been done (cf. [T2, pp. 641-642]). We do it again here by a different method because some of the intermediate lemmas will be needed later in the paper.

(3.14) LEMMA. Let $f(x, y) \in \mathbf{C}[[x, y]]$ be the equation of a reduced algebroid plane curve. Then there exists an integer $l$ (dependent on $f$ ) such that for all $g(x, y)$ in the ideal $(x, y)^{l}$ the family of algebroid plane curves $f(x y)+t g(x, y)$ over Spec $\mathbf{C}[[t]]$ is a trivial deformation.

ProOF. To show that $f(x, y)+t g(x, y)$ is a trivial deformation we must find power series $A(x, y, t)=x+a_{1}(x, y) t+a_{2}(x, y) t^{2}+\cdots, B(x, y, t)=y+b_{1}(x, y) t$ $+b_{2}(x, y) t^{2}+\cdots$, and $C(x, y, t)=1+c_{1}(x, y) t+c_{2}(x, y) t^{2}+\cdots$ such that $f(A, B)+$ $\operatorname{tg}(A, B)=C f(x, y)$. First we write both sides of this equation as power series in $t$. 


$$
\begin{gathered}
f(x, y)+t\left(\frac{\partial f}{\partial x}(x, y) a_{1}(x, y)+\frac{\partial f}{\partial y}(x, y) b_{1}(x, y)+g(x, y)\right) \\
+t^{2}\left(\frac{\partial f}{\partial x}(x, y) a_{2}(x, y)+\frac{\partial f}{\partial y}(x, y) b_{2}(x, y)+\frac{\partial^{2} f}{\partial x^{2}}(x, y) a_{1}(x, y)^{2}\right. \\
+2 \frac{\partial^{2} f}{\partial x \partial y}(x, y) a_{1}(x, y) b_{1}(x, y)+\frac{\partial^{2} f}{\partial y^{2}}(x, y) b_{1}(x, y)^{2} \\
\left.+\frac{\partial g}{\partial x}(x, y) a_{1}(x, y)+\frac{\partial g}{\partial y}(x, y) b_{1}(x, y)\right) \\
+\cdots+t^{n}\left(\frac{\partial f}{\partial x}(x, y) a_{n}(x, y)+\frac{\partial f}{\partial y}(x, y) b_{n}(x, y)+T_{n}\right)+\cdots \\
=f(x, y)+t c_{1}(x, y) f(x, y)+t^{2} c_{2}(x, y) f(x, y)+\cdots+t^{n} c_{n}(x, y) f(x, y)+\cdots
\end{gathered}
$$

Here

$$
\begin{aligned}
T_{n}= & \frac{\partial g}{\partial x}(x, y) a_{n-1}(x, y)+\frac{\partial g}{\partial y}(x, y) b_{n-1}(x, y) \\
& +\left(\text { terms which have no } a_{i} \text { 's or } b_{i} \text { 's with } i \geq n,\right. \\
& \left.\quad \text { and have at least two factors that are } a_{i} \text { 's or } b_{i} \text { 's, } i<n\right) .
\end{aligned}
$$

We know that there exists an integer $k$ such that the Jacobian ideal of $f$ contains all monomials of degree greater than or equal to $k$. Choose $l=2 k$. We now explain inductively how to choose the $a_{i}, b_{i}$, and $c_{i}$.

$i=1$. Because $g(x, y) \in(x, y)^{2 k}$ we may find a relation

$$
g(x, y)=a(x, y) \frac{\partial f}{\partial x}+b(x, y) \frac{\partial f}{\partial y}+c(x, y) f
$$

with $a, b, c \in(x, y)^{k}$. (Use the fact that $(x, y)^{k} \cdot(x, y)^{k}=(x, y)^{2 k}$ and the Jacobian contains all monomials of degree $\geq k$.) Set $a_{1}=-a, b_{1}=-b, c_{1}=c$.

Having chosen up to $n-1$ choose for $n$ as follows. Recall that all the $a_{i}, b_{i}$, and $c_{i}$ for $i \leq n-1$ are in $(x, y)^{k}$. Thus, $T_{n} \in(x, y)^{2 k}$ and we may find a relation

$$
T_{n}=\alpha(x, y) \frac{\partial f}{\partial x}+\beta(x, y) \frac{\partial f}{\partial y}+\gamma(x, y) f
$$

with $\alpha, \beta, \gamma \in(x, y)^{k}$. Set $a_{n}=-\alpha, b_{n}=-\beta, c_{n}=\gamma$.

(3.15) LEMMA. Let $f(x, y)+t^{m_{1}} g_{m_{1}}(x, y)+\cdots$ be the equation of a flat family of reduced algebroid plane curves over $\operatorname{Spec} \mathbf{C}\left[\left[t^{m_{1}}, \ldots, t^{m_{k}}\right]\right]=: Z$. Via the inclusion $\mathbf{C}\left[\left[t^{m_{1}}, \ldots, t^{m_{k}}\right]\right] \subset \mathbf{C}[[t]]$ this is also the equation of a flat family of reduced algebroid plane curves over Spec $\mathbf{C}[[t]]=: Y$. The family over $Z$ is trivial if and only if the family over $Y$ is trivial.

ProOF. Clearly if the family over $Z$ is trivial then the family over $Y$ is trivial. Now suppose the family over $Y$ is trivial. This means we have power series $A(x, y, t)$, $B(x, y, t), C(x, y, t)$ as in the proof of (3.14) with

$$
f(A, B)+t^{m_{1}} g_{m_{1}}(A, B)+\cdots=C f(x, y) .
$$

To show that this actually gives a trivialization over $Z$ one must show that $A, B$, and $C$ do not contain any powers of $t$ not allowed in $Z$. Expand both sides of (3.16) 
in powers of $t$. Using the fact that

$$
\begin{aligned}
\frac{\partial^{n} f}{\partial t^{n}}=\frac{\partial f}{\partial x} & \frac{\partial^{n} x}{\partial t^{n}}+\frac{\partial f}{\partial y} \frac{\partial^{n} y}{\partial t^{n}}+\text { terms of the form } \\
& \text { (some higher partial of } f)\left(\left(\frac{\partial^{p} x}{\partial t^{p}}\right)^{i}\left(\frac{\partial^{q} y}{\partial t^{q}}\right)^{j}\right) \text { with } i p+j q=n
\end{aligned}
$$

one may deduce that no unwanted powers of $t$ occur.

(3.17) REMARK. Notice that (3.15) remains true if we replace $\mathbf{C}[[t]]$ and $\mathbf{C}\left[\left[t^{m_{1}}, \ldots, t^{m_{k}}\right]\right]$ by $\mathbf{C}[[t]] /\left(t^{n}\right)$ and $\mathbf{C}\left[\left[t^{m_{1}}, \ldots, t^{m_{k}}\right]\right] /\left(t^{n}\right)$.

(3.18) Proposition. Let $f: X \rightarrow Y$ be a flat family of reduced algebroid plane curves, where $Y$ is as in the statement of the versal property of the formal versal deformation. Assume the family is trivial. Then there is a unique morphism from $Y$ to $B^{\prime}$ (the base of the formal versal deformation) which induces $f: X \rightarrow Y$. This morphism is the obvious one sending all of $Y$ to the closed point of $B^{\prime}$.

ProOF. Otherwise, using (3.15) and (3.17), one may deduce that there would be a subscheme $Z$ of $B^{\prime}$ larger than the reduced point $(0, \ldots, 0)$ over which the versal family is trivial. This contradicts the fact that this versal family may be taken to be a universal first order deformation.

(3.19) COROllary. Suppose that $f(x, y)$ and $g(x, y)$ in (3.14) are polynomials. In this situation we may think of $f+t g$ as a flat family of curves in $\mathbf{A}_{x, y}^{2}$ parametrized by $\mathbf{A}_{t}^{1}$. If $g$ is chosen as in (3.14) then for all but finitely many values of $t$ the curve $f+t g=0$ has a singularity analytically isomorphic to the singularity of $f$ at $(0,0)$. In fact an étale neighborhood of this singularity is isomorphic to an étale neighborhood of $(0,0)$ in $f=0$.

ProOF. An étale neighborhood of $0 \in \mathbf{A}_{t}^{1}$ maps to an étale neighborhood of the origin $(0, \ldots, 0) \in B$ (the base of the étale versal deformation). There is an induced morphism from the spectrum of the complete local ring of $0 \in A_{t}^{1}$ to the spectrum of the complete local ring of $(0, \ldots, 0) \in B$. This morphism must be a morphism one gets by thinking of $f+t g$ as a family of algebroid plane curves over $\operatorname{Spec} \mathbf{C}[[t]]$ and thereby obtaining from the versal property a morphism Spec $\mathbf{C}[[t]] \rightarrow B^{\prime}$. By (3.14) and (3.18) the morphism Spec $\mathbf{C}[[t]] \rightarrow B^{\prime}$ maps everything to the closed point. Thus the original morphism on étale neighborhoods maps everything to $(0, \ldots, 0)$.

(3.20) Corollary. Let $D_{1}, \ldots, D_{n}$ be reduced curves in $\mathbf{P}^{2}$ and $p_{i} \in D_{i}$ singular points. Then for any sufficiently large integer $e$ there exists a reduced irreducible curve $E$ in $\mathbf{P}^{2}$ of degree $e$ such that $E$ has exactly $n$ singular points $q_{1}, \ldots, q_{n}$ and for each $i$ an étale neighborhood of $q_{i}$ in $E$ is isomorphic to an étale neighborhood of $p_{i}$ in $D_{i}$.

PROOF. We may assume that the $D_{i}$ have no common components and $p_{i} \notin D_{j}$ for $i \neq j$. Let $d_{i}$ be the degree of $D_{i}$ and $d$ the sum of the $d_{i}$. Assume that $D_{i}=\left\{F_{i}\left(X_{0}, X_{1}, X_{2}\right)=0\right\}$ and that $p_{i} \notin\left\{X_{0}=0\right\}$ for all $i$. For any integer $e \gg 0$ we may find a curve $E_{1}=\left\{G\left(X_{0}, X_{1}, X_{2}\right)=0\right\}$ of degree $e \geq d$ with the following properties. 
(1) One may choose affine coordinates $x_{i}, y_{i}$ on the affine patch $X_{0} \neq 0$ with $p_{i}=(0,0)$ such that if $f_{i}\left(x_{i}, y_{i}\right) \in \mathbf{C}\left[x_{i}, y_{i}\right]$ is the equation for $D_{i}$ and $g_{i}\left(x_{i}, y_{i}\right) \in$ $\mathrm{C}\left[x_{i}, y_{i}\right]$ is the equation for $E_{1}$ in these coordinates then in (3.14) $f_{i}$ may replace $f$ and $g_{i}$ will will satisfy the conditions placed on $g$.

(2) $E_{1}$ is nonsingular away from $\bigcup_{i=1}^{n} p_{i}$, and irreducible.

Consider the pencil of plane curves $\mu X_{0}^{e-d} \prod_{i=1}^{n} F_{i}+\lambda G=0,[\mu, \lambda] \in \mathbf{P}^{1}$. By Bertini's theorem a general element of this pencil is nonsingular away from $\bigcup_{i=1}^{n} p_{i}$ and irreducible. By (3.19) a general element of this pencil will have the desired singularities at the $p_{i}$ 's.

(3.21) LEMMA. Let $D=\{f(x, y)=0\}$ be an affine plane curve whose only singularity is at $(0,0)$. Construct the étale versal deformation of $D$ as at the beginning of this section. Then there exists a Zariski open subset $U$ of $B$ containing $(0, \ldots, 0)$ on which $(0, \ldots, 0)$ is the only point whose fiber has a singularity analytically isomorphic to the singularity of $D$ at $(0,0)$.

PROOF. Let $Z$ be the set of points of $B$ whose fibers have a singularity analytically isomorphic to the singularity of $D$ at $(0,0)$. We first show that $Z$ is Zariski constructible.

For any fixed values of $t_{1}, \ldots, t_{m}, f(x, y)+\sum t_{i} g_{i}=0$ has a singularity analytically isomorphic to the singularity of $D$ at $(0,0)$ if and ony if there exist power series

$$
X=\sum_{i, j \geq 0} a_{i j} x^{i} y^{j}, \quad Y=\sum_{i, j \geq 0} b_{i j} x^{i} y^{j}
$$

with

$$
\operatorname{rank}\left[\begin{array}{cc}
a_{10} & a_{01} \\
b_{10} & b_{01}
\end{array}\right]=2
$$

and a constant $c$ such that

$$
f(X, Y)+\sum t_{i} g_{i}(X, Y)=c f(x, y) .
$$

Expanding both sides of (3.22) as power series in $x$ and $y$ and comparing coefficients of terms of the same degree we at first appear to have infinitely many constraints on the $t_{i}$ 's. However, from (3.14) and (3.19) or equivalently the fact that plane algebraic curve singularities are finitely determined we see that we need only check that (3.22) holds up to a fixed finite power in $x$ and $y$. This shows that $Z$ is Zariski constructible.

Because $Z$ is Zariski constructible, if there did not exist a $U$ as desired we could find a curve through $(0, \ldots, 0)$ along which $(3.22)$ was satisfied. At $(0, \ldots, 0)$ the versal family restricted to this curve would be formally locally trivial. This contradicts the fact that the formal versal deformation can be taken to be a universal first order deformation.

(3.23) Proposition. Consider a flat family of reduced curves in $\mathbf{A}^{2}$.

$$
\begin{aligned}
& X \subset Y \times \mathbf{A}^{2} \\
& \downarrow p=\text { projection } \\
& Y
\end{aligned}
$$

Assume that $Y$ is reduced, separated, and of finite type over C. Let $y$ be a closed point of $Y$ and $x$ a singular point of $p^{-1}(y)$. Then the following statements are equivalent. 
(1) There exists a Zariski open set $U \subset X$ with $x \in U$ such that the restricted family $p: U \rightarrow p(U)$ is formally locally trivial at $y$.

(2) There exists a Zariski open set $U \subset X$ with $x \in U$ such that the restricted family $p: U \rightarrow p(U)$ is formally locally trivial.

(3) For each Zariski open neighborhood $U$ of $x$ in $X$ there exists a Zariski open neighborhood $U^{\prime} \subset U$ of $x$ in $X$ such that for all closed points $z \in p\left(U^{\prime}\right), p^{-1}(z) \cap U^{\prime}$ has only one singularity and it is analytically isomorphic to the singularity of $f^{-1}(y)$ at $x$.

(4) There exist étale neighborhoods $V$ of $y$ in $Y$ and $W$ of $x$ in $V \times_{Y} X$ such that the induced family $p: W \rightarrow V$ is trivial.

ProOF. (4) $\Rightarrow(2)$ The image of $W$ in $X$ must contain a Zariski open neighborhood $U$ of $x$ in $X$. This is the desired $U$.

$(2) \Rightarrow(1)$ Obvious.

$(1) \Rightarrow(4)$ Reason as in the proof of (3.19).

(4) $\Rightarrow$ (3) $W$ may be chosen small enough so that $W \cap p^{-1}(y)$ has $x$ as its only singular point. The image of $W$ in $X$ must contain a Zariski open neighborhood $U$ of $x$ in $X$. This is the desired $U$.

$(3) \Rightarrow(4)$ Using (3.20) we know there is a plane curve $D$ whose only singularity has an étale neighborhood isomorphic to an étale neighborhood of $x$ in $f^{-1}(y)$. Using this $D$ construct the étale versal deformation $\pi: \mathscr{C} \rightarrow B$. An étale neighborhood $V$ of $y$ in $Y$ maps to an étale neighborhood of $(0, \ldots, 0) \in B$. Since we are assuming (3), (3.21) says that all of $V$ maps to $(0, \ldots, 0)$. This says the family $V \times_{Y} X \rightarrow V$ is trivial.

(3.24) REMARKS. (a) In the statement of (3.23) one could replace $\mathbf{A}^{2}$ with any nonsingular surface of finite type over $\mathbf{C}$ and the proposition would remain true. To see this let $D$ be any reduced separated curve of finite type over $\mathbf{C}$ and $p$ a singular point of $D$. Assume that $D$ may be embedded in a nonsingular surface of finite type over $\mathbf{C}$. An open neighborhood of $p$ in $D$ embeds in some affine space $\mathbf{A}^{n}$. Since $D$ embeds in a nonsingular surface the tangent space to $D$ at $p$ is two dimensional. This says that a generic projection from $\mathbf{A}^{n}$ to $\mathbf{A}^{2}$ embeds a neighborhood of $p$ in $D$ as a subscheme of $\mathbf{A}^{2}$.

(b) In the statement of (3.23) we cannot replace (3) with the simpler statement: "There exists a Zariski open set $U \subset X$ with $x \in U$ such that for all closed points $z \in p(U), p^{-1}(z) \cap U$ has only one singularity and it is analytically isomorphic to the singularity of $f^{-1}(y)$ at $x$." A counterexample may be constructed as follows. Let $f: X \rightarrow Y$ be a family of curves where the general fiber has one node but the fiber over $y \in Y$ has two nodes. Call the node on $f^{-1}(y)$ that is not a limit of nodes on general fibers $x$ and call the node which is $z$. Find a divisor $D$ on $X$ which meets $f^{-1}(y)$ at $z$ and perhaps other points but not $x$ and meets a general fiber at nonsingular points. Set $U=X-D$. This family now satisfies the proposed alternate for (3) but not any of (1)-(4).

We now investigate the relationship between equisingular families and families in which all fibers over closed points have equivalent singularities. This has been previously done when the base of the family is regular (cf. [T2, pp. 622-624]), but we wish to consider families over any reduced separated finite type base.

(3.25) THEOREM [W, pp. 144,158,164]. (1) Let $f \in \mathbf{C}[[x, y]]$ be the equation of a reduced algebroid plane curve. Let $\mathbf{C}[\varepsilon]=\mathbf{C}[\varepsilon] /\left(\varepsilon^{2}\right)$ be the ring of dual numbers. 
set $I=\{g \in \mathbf{C}[[x, y]]: f+\varepsilon g$ is the equation of an equisingular family of algebroid plane curves over Spec $\mathbf{C}[\varepsilon]\}$. Then I is an ideal and I contains the Jacobian ideal of $f$. I is called the equisingular ideal of $f$.

(2) (Recall that we denote by $B^{\prime}$ the base of the formal versal deformation of the singularity of $f$.) There exists a smooth closed subscheme $E S$ of $B^{\prime}$ on which the induced family of curves yields a formal versal equisingular deformation of the singularity of $f$.

(3) Under the identification $B^{\prime}=\mathrm{C}[[x, y]] / J$ the tangent space to $E S$ at the origin is identified with $I / J$.

(3.26) REMARK. As defined, $I$ is an ideal in $\mathrm{C}[[x, y]]$. We will also denote by $I$ the ideals obtained from $I$ by extension and/or contraction in other appropriate rings such as $\mathbf{C}[x, y]$ or the local ring of the curve in question at the singular point in question.

(3.27) ThEOREM $[\mathbf{T 2}, \S 3,5]$. Let $D$ be a reduced curve in $\mathbf{A}^{2}$ whose unique singularity is at $(0,0)$. Construct the étale versal deformation $\pi: \mathscr{C} \rightarrow B$ for $D$ as at the beginning of this section. Define $D_{\mu}=\left\{p \in B: \pi^{-1}(p)\right.$ is a reduced curve with only one singularity and that singularity is equivalent to the singularity of $D$ at $(0,0)\} . D_{\mu}$ has the following properties. (Use the standard metric topology on $\mathbf{C}^{n}$.)

(1) In some open subset of $B$ containing $(0, \ldots, 0) D_{\mu}$ is a closed analytic subvariety of $B$.

(2) $D_{\mu}$ is smooth at $(0, \ldots, 0)$ and under the identification $B=\mathbf{C}[x, y] / J$ the tangent space to $D_{\mu}($ at $(0, \ldots, 0))$ is $I / J$.

(3) In some open subset of $B$ containing $(0, \ldots, 0)$ the family $\pi^{-1}\left(D_{\mu}\right) \rightarrow D_{\mu}$ is equisingular.

(3.28) Lemma. Let $D=\{f(x, y)=0\}$ be a reduced curve in $\mathbf{A}^{2}$. Assume that $(0,0)$ is the only singular point of $D$. Let $B=\operatorname{Spec} \mathbf{C}\left[t_{1}, \ldots, t_{m}\right]$ and $B^{\prime}=$ Spec $\mathbf{C}\left[\left[t_{1}, \ldots, t_{m}\right]\right]$ be the bases of the étale and formal versal deformation spaces of the singularity of $D$ at $p$ as constructed at the beginning of this section. The natural inclusion $\mathbf{C}\left[t_{1}, \ldots, t_{m}\right] \rightarrow \mathbf{C}\left[\left[t_{1}, \ldots, t_{m}\right]\right]$ induces a dominant morphism $\psi:$ Spec $\mathbf{C}\left[\left[t_{1}, \ldots, t_{m}\right]\right] \rightarrow \operatorname{Spec} \mathbf{C}\left[t_{1}, \ldots, t_{m}\right]$. Let $Z$ be the closure of $\psi(E S)$.

(1) There exists a Zariski open subset $U$ of $Z$ containing $(0, \ldots, 0)$ over which the étale versal family is equisingular.

(2) Under the identification $B=\mathbf{C}[x, y] / J$ the tangent space to $U$ at $(0, \ldots, 0)$ is identified with $I / J$. In particular $U$ is nonsingular at $(0, \ldots, 0)$.

PROOF. One would hope that this would follow easily from (3.25).

The equimultiple sections of the family over $E S$ that show that family is equisingular give equimultiple sections over some Zariski neighborhood of $(0, \ldots, 0)$ in $Z$ of the family over $Z$. This proves (1).

From (3.25) (3) we see that the tangent space to $U$ at $(0, \ldots, 0)$ is at least $I / J$. Part (1) of this lemma implies that the formal versal family is equisingular over $\psi^{-1}(U)$. Together with (3.25) (3) this implies that the tangent space to $U$ at $(0, \ldots, 0)$ cannot be any larger than $I / J$.

(3.29) Definition. We will call the open set $U$ of (3.28) ES. 
(3.30) Proposition. Consider a flat family of reduced curves in $\mathbf{A}^{2}$.

$$
\begin{aligned}
& X \subset Y \times \mathbf{A}^{2} \\
& \downarrow p=\text { projection } \\
& Y
\end{aligned}
$$

Assume that $Y$ is reduced, separated, and of finite type over $\mathbf{C}$. Let $y$ be a closed point of $Y$ and $x$ a singular point of $p^{-1}(y)$. Then the following statements are equivalent.

(1) For each Zariski open neighborhood $U$ of $x$ in $X$ there exists a Zariski open neighborhood $U^{\prime} \subset U$ of $x$ in $X$ such that for all closed points $z \in p\left(U^{\prime}\right), p^{-1}(z) \cap U^{\prime}$ has only one singularity and it is equivalent to the singularity of $f^{-1}(y)$ at $x$.

(2) There exist étale neighborhoods $V$ of $y$ in $Y$ and $W$ of $x$ in $V \times_{Y} X$ such that the induced family $p: W \rightarrow V$ is equisingular.

ProOF. $(2) \Rightarrow(1)$ The fact that the family is equisingular implies the existence of a sequence of blowups with certain properties. Restricted to each fiber this provides a sequence of blowups to show that their singularities are equivalent. $W$ may be chosen small enough so that $W \cap p^{-1}(y)$ has $x$ as its only singular point. The image of $W$ in $X$ must contain a Zariski open neighborhood $U$ of $x$ in $X$. This is the desired $U$.

$(1) \Rightarrow(2)$ Using (3.20) we know there is a plane curve $D$ whose only singularity has an étale neighborhood isomorphic to an étale neighborhood of $x$ in $f^{-1}(y)$. Using this $D$ construct the étale versal deformation $\pi: \mathscr{C} \rightarrow B$. An étale neighborhood $V$ of $y$ in $Y$ maps to an étale neighborhood of $(0, \ldots, 0) \in B$. Since we are assuming (1), using (3.25), (3.27), and (3.28) we conclude that after possibly shrinking $V$ to $V^{\prime}$ the image of $V^{\prime}$ lies in $E S$. This says the family $V^{\prime} \times_{Y} X \rightarrow V^{\prime}$ is equisingular.

The remarks in (3.24) also apply to (3.30).

(3.31) PROPOSITION. Consider a flat family of reduced curves on a nonsingular surface $S$ separated of finite type over $\mathbf{C}$.

$$
\begin{aligned}
& X \subset Y \times S \\
& \downarrow p=\text { projection } \\
& Y
\end{aligned}
$$

Assume that $Y$ is reduced, separated, and of finite type over $\mathbf{C}$. Let $y$ be a closed point of $Y$ and $x$ a singular point of $p^{-1}(y)$. Then given any étale neighborhoods $U$ of $y$ and $V$ of $x$ in $p^{-1}(U)$ after possibly shrinking $U$ to $U^{\prime}$ and $V$ to $V^{\prime}$ one may obtain a family $V^{\prime} \rightarrow U^{\prime}$ on which the function $\delta: U^{\prime} \rightarrow \mathbf{Z}$ of (3.13)(5) is upper semicontinuous.

PROOF. It is enough to show that this is true in the étale versal deformation of any reduced curve $D$ with singular point $p$ such that an étale neighborhood of $p$ in $D$ is isomorphic to an étale neighborhood of $x$ in $p^{-1}(y)$. By (3.20) we may choose $D=\{f(x, y)=0\} \subset \mathbf{A}^{2}, p=(0,0)$, where $f$ is the dehomogenization of a form $F\left(x_{0}, x_{1}, x_{2}\right)$ where $F=0$ is a reduced irreducible curve in $\mathbf{P}^{2}$ with only one singular point. Furthermore we may choose $f$ to be of sufficiently high degree so that the $g_{i}(x, y)$ we choose to construct the étale versal deformation all have degree less than or equal to $f$. Say $g_{i}(x, y)$ is the dehomogenization of $G_{i}\left(x_{0}, x_{1}, x_{2}\right), x_{0}=0$ 
is the line at infinity, and $k_{i}=\operatorname{degree} F-\operatorname{degree} G_{i}$. Then $F+\sum t_{i} x_{0}^{k_{i}} G_{i}=0$ gives a family of projective plane curves over $\mathbf{A}_{t_{1}, \ldots, t_{m}}^{m}$. Remove all points lying below nonreduced curves and all points corresponding to curves with singularities on $x_{0}=0$. Recall that the geometric genus of a reduced curve $E$ of degree $d$ in $\mathbf{P}^{2}$ is $\frac{1}{2}(d-1)(d-2)-\delta(E)$. Since in this family all fibers have the same degree, $(2.4)$ completes the proof.

(3.32) Proposition. Let

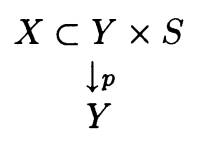

be a flat family of reduced curves on a smooth surface $S$. Assume that $X, Y$, and $S$ are all reduced, separated, and of finite type over $\mathbf{C}$. Let $\Delta$ be the locus of singular points of fibers of $p$. Assume that $\Delta$ is proper over $Y$. Then the following two conditions are equivalent.

(1) The family is locally equisingular in the étale topology.

(2) For each equivalence class of singularity all fibers over closed points of $Y$ have the same number of singularities of that equivalence class.

PROOF. (1) $\Rightarrow$ (2) follows easily from (3.30) and (3.24).

(2) $\Rightarrow(1)$ The locus $\Delta$ is the locus where the sheaf of relative differentials fails to be locally free; thus $\Delta$ is a closed subset of $X$. Since $\Delta$ is proper over $Y$ we see that every component of $\Delta$ either surjects onto $Y$ or maps to a proper closed subvariety of $Y$.

Suppose there is a proper closed subvariety $R$ of $Y$ over which there lies a component of $\Delta$ whose image in $Y$ is $R$. Pick a closed point $r \in R$. On $p^{-1}(r)$ we have two types of singular points: (a) those which are limits of nearby singular points on fibers over $Y-R$, and (b) the other "new" singular points. Observe that if $s$ and $t$ are equivalent singularities then $\delta(s)=\delta(t)$. Using assumption (2) we conclude that there exists an integer $d$ such that $\delta\left(f^{-1}(y)\right)=d$ for all closed $y \in Y$. Using (3.31) we conclude that the sum $\sum \delta(s)$, where $s$ runs over all singular points of type (a) on $p^{-1}(r)$, is greater than or equal to $d$. This means there can be no singularities of type (b) - a contradiction to the existence of $R$. Thus, every component of $\Delta$ surjects onto $Y$.

Again using the assumptions of (2) we see that locally in the analytic topology $\Delta$ consists of set theoretic sections of $p$. In order to keep the total number of singularities of each equivalence type constant from fiber to fiber any change in the equivalence type of singularity along one section would have to be compensated for by changes on other sections. This is impossible because different equivalence types of singularities cannot be simultaneously degenerations of each other. (Think about what would have to happen in the étale versal deformation spaces.) We conclude that the equivalence class of singularity is constant along each section.

Locally in the étale topology this family must be obtained via pull back from the étale versal deformation. Using (3.27) we conclude that this family is locally equisingular in the analytic topology and the local set theoretic sections are actually local analytic sections. Finally since $\Delta$ is a closed algebraic subset of $X$ we see that the local analytic sections are local étale sections and the family is locally equisingular in the étale topology. 
(3.33) Definition. Let $D=\{f(x, y)=0\} \subset \mathbf{A}^{2}$ be a reduced curve whose only singularity is at $(0,0)$. Construct the étale and formal versal deformation spaces for $D$ as at the beginning of this section. Recall the functions $\delta$ and $\kappa$ of (3.12) and (3.13)(5). Define

$$
\begin{aligned}
E G & =\{p \in B: \delta(p)=\delta(0, \ldots, 0)\} \\
E G^{\prime} & =\left\{p \in B^{\prime}: \delta(p)=\delta(0, \ldots, 0)\right\} \\
E C & =\{p \in B: \delta(p)=\delta(0, \ldots, 0) \text { and } \kappa(p)=\kappa(0, \ldots, 0)\} \\
E C^{\prime} & =\left\{p \in B^{\prime}: \delta(p)=\delta(0, \ldots, 0) \text { and } \kappa(p)=\kappa(0, \ldots, 0)\right\} .
\end{aligned}
$$

In the notation of (3.28) $E G^{\prime}=\psi^{-1}(E G)$ and $E C^{\prime}=\psi^{-1}(E C)$. Proposition (3.31) tells us that in some Zariski open neighborhood of $(0, \ldots, 0) E G$ is closed and therefore $E G^{\prime}$ is closed. The same will be shown for $E C$ and $E C^{\prime}$ in (4.18).

4. Tangent spaces and tangent cones. Let

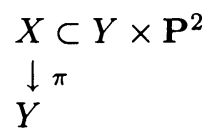

be a flat equigeneric family of reduced projective plane curves. Assume that $Y$ is irreducible, nonsingular, separated, and of finite type over C. Let $h: X^{\prime} \rightarrow X$ be the normalization map. By (2.5) $\pi \circ h: X^{\prime} \rightarrow Y$ is a smooth family of reduced projective curves. We shall define two ideal sheaves on $X^{\prime}$, first abstractly as Fitting ideals and then in terms of local coordinates. Our Fitting ideals are numbered as in [ACGH, p. 179].

(4.1) Definition. $\mathcal{J}$ is defined to be the pull back to $X^{\prime}$ of the dimension $(Y+2)$ th Fitting ideal of the sheaf of differentials on $X$. $\mathscr{R}$ is defined to be the dimension $(Y+1)$ th Fitting ideal of the sheaf of relative differentials of $X^{\prime}$ over $X$.

Choose an open subset $U$ of $Y \times \mathbf{P}^{2}$ on which we may choose $x, y$ as local coordinates on $\mathbf{P}^{2}, t_{1}, \ldots, t_{m}$ local coordinates on $Y$, and $F\left(t_{1}, \ldots, t_{m}, x, y\right)$ the local equation for $X$. Furthermore suppose that on the inverse image of $U$ in $X^{\prime}$ we may choose $z$ to be a local coordinate along the fibers of $\pi \circ h$ so that on $X x=x\left(z, t_{1}, \ldots, t_{m}\right), y=y\left(z, t_{1}, \ldots, t_{m}\right)$. Then on the inverse image of $U$ in $X^{\prime}$ (or possibly some smaller open set) we have that the ideal $\mathcal{J}$ is generated by $(\partial F / \partial x, \partial F / \partial y)$ and the ideal $\mathscr{R}$ is generated by $(\partial x / \partial z, \partial y / \partial z)$.

For any point $y \in Y$ let $X_{y}$ and $X_{y}^{\prime}$ denote the fibers of $\pi$ and $\pi \circ h$ over $y . X_{y}^{\prime}$ is a nonsingular curve. Denote by $\mathscr{J}_{y}$ and $\mathscr{R}_{y}$ the effective divisors on $X_{y}^{\prime}$ obtained by restricting the ideals $\mathscr{J}$ and $\mathscr{R}$ to $X_{y}^{\prime}$. Also denote by $\mathscr{A}_{y}$ the effective divisor on $X_{y}^{\prime}$ given by the pull back of the conductor of $X_{y}$ to $X_{y}^{\prime}$. From [P, p. 261] we see that we have the relation

$$
\mathscr{A}_{y}=\mathscr{J}_{y}-\mathscr{R}_{y} .
$$

We are assuming that the family is equigeneric. This means that $\mathscr{A}_{y}$ must have the same degree for all $y$. Together with (4.2) this implies that there exists a (Cartier) divisor $A^{\prime}$ on $X^{\prime}$ such that $A^{\prime}$ restricts to $\mathscr{A}_{y}$ on $X_{y}^{\prime}$ for all $y$.

Notice also that $m\left(X_{y}\right)=\operatorname{degree}\left(\mathscr{R}_{y}\right)$ (see (3.12)). This shows that $\kappa\left(X_{y}\right)$ is an upper semicontinuous function on $Y$. Also if we assume further that the family $\pi: X \rightarrow Y$ is equiclassical then $\mathscr{R}$ must describe a (Cartier) divisor $R^{\prime}$ on $X^{\prime}$ 
and by (4.2) $\mathcal{J}$ describes a (Cartier) divisor $J^{\prime}$ on $X^{\prime}$, and we have the relation $A^{\prime}=J^{\prime}-R^{\prime}$.

(4.3) Definition. Assume that $Y$ is a single point so that $X$ is a single curve. Let $H$ be the ideal sheaf given by $H(U)=\left\{g \in \mathscr{O}(U): h^{*} g \in \mathscr{J}\right\}$. If $p$ is a point of $X$ then the ideal in the local ring of $X$ at $p$ obtained from $H$ is called the equiclassical ideal and is also denoted by $H$.

Continue to assume that $Y$ is a single point. Notice that $\mathscr{J}=h^{*} J(J$ is the Jacobian ideal). For an open set $U \subset X, H(U)=\{g \in \mathscr{O}(U)$ : the divisor $\left.\left(h^{*} g\right) \geq A^{\prime}+R^{\prime}=J^{\prime}\right\}$.

(4.4) LEMMA. Let

$$
\begin{aligned}
& X \subset \operatorname{Spec} \mathbf{C}[[t]] \times \operatorname{Spec} \mathbf{C}[[x, y]] \\
& \downarrow \pi \\
& \text { Spec } \mathbf{C}[[t]]
\end{aligned}
$$

be an equigeneric flat family of reduced algebroid plane curves with equation

$$
0=F(t, x, y)=f(x, y)+t^{m} f_{m}(x, y)+t^{m+1} f_{m+1}(x, y)+\cdots .
$$

Then $f_{m}(x, y)$ is in the conductor ideal of the singularity defined by $f(x, y)=0$. If furthermore the family is equiclassical then $f_{m}(x, y)$ is in the equiclassical ideal of the singularity defined by $f(x, y)=0$.

PROOF. First we recall one description of the conductor ideal. Let $X_{0}$ be a reduced plane curve. Let $h: X_{0}^{\prime} \rightarrow X_{0}$ be the normalization map. Then the conductor $A$ of $X_{0}$ is the ideal $\operatorname{Hom}_{\mathscr{O}_{X_{0}}}\left(h_{*} \mathscr{O}_{X_{0}^{\prime}}, \mathscr{O}_{X_{0}}\right)$ (cf. [P, p. 261]). From this description it is easy to see that $g \in A$ if and only if $h^{*} g \in h^{*} A$.

Our family $\pi: X \rightarrow \operatorname{Spec} \mathbf{C}[[t]]$ can be obtained via base change from the formal versal deformation, and the formal versal deformation is obtained via taking completions from the étale versal deformation which is a family of finite type. Therefore what we have said from the beginning of $\S 4$ up to (4.4) also applies to this family. Continue with the notation of that discussion.

What we need to show is that, restricted to the fiber $t=0$ of $\pi \circ h,\left(f_{m}\right) \geq A^{\prime}$. It is sufficient to show this separately on each branch, so let $E=\operatorname{Spec} \mathbf{C}[[z]] \times \operatorname{Spec} \mathbf{C}[[t]]$ be some fixed family of normalized branches. Let $\mathscr{I}\left(A^{\prime}\right)$ be the ideal of $A^{\prime}$. (4.2) says

$$
\mathscr{I}\left(A^{\prime}\right) \supset \mathscr{J} \text {. }
$$

On $E$ the function $F(t, x, y)$ is identically zero. This gives

$$
0=\frac{d F}{d t}=\frac{\partial F}{\partial x} \frac{d x}{d t}+\frac{\partial F}{\partial y} \frac{d y}{d t}+\frac{\partial F}{\partial t}
$$

which means $\partial F / \partial t \in \mathcal{J}$ and (4.5) implies the following relation between divisors:

$$
(\partial F / \partial t) \geq A^{\prime} .
$$

Expand $\partial F / \partial t$ in powers of $t$ :

$$
\frac{\partial F}{\partial t}=m t^{m-1} f_{m}(x, y)+(m+1) t^{m} f_{m+1}(x, y)+\cdots .
$$


This says $(\partial F / \partial t)=(m-1)(t)+F^{\prime}, F^{\prime}$ effective. Since $A^{\prime}$ does not have $(t)$ as a component (4.7) says $F^{\prime} \geq A^{\prime}$. In other words

$$
\left(m f_{m}(x, y)+(m+1) t f_{m+1}(x, y)+\cdots\right) \geq A^{\prime} .
$$

Set $t=0$ and conclude that on the fiber $t=0$ of $\pi \circ h$ either $f_{m}$ vanishes identically or $\left(f_{m}\right) \geq A^{\prime}$, so $f_{m} \in A$.

Now assume further that the family is equiclassical. Now (4.6) says

$$
(\partial F / \partial t) \geq J^{\prime} .
$$

Following the same reasoning as before we show that on the fiber $t=0$ of $\pi \circ h$ either $f_{m}$ vanishes identically or $\left(f_{m}\right) \geq J^{\prime}$, so $f_{m} \in H$.

(4.8) Definition. Suppose we have $D \subset U=\operatorname{Spec} R$ where $D$ is either a reduced algebroid plane curve and $R=\mathbf{C}[[x, y]]$ or $D$ is a reduced curve on $U=$ an affine open subset of $\mathbf{P}^{2}$. Let $f$ be an equation for $D$. Let

$$
\begin{aligned}
& X \subset Y \times U \\
& \downarrow \pi \\
& y \in Y
\end{aligned}
$$

be a flat deformation of $D$ as a subscheme of $U$, the fiber over $y$ being the given embedding of $D$ in $U$. If $h$ : Spec $\mathbf{C}[\varepsilon] /\left(\varepsilon^{2}\right) \rightarrow Y$ is a morphism whose image contains $y$ then the pull back family over Spec $\mathbf{C}[\varepsilon] /\left(\varepsilon^{2}\right)$ will have in $U \times \operatorname{Spec} \mathbf{C}[\varepsilon] /\left(\varepsilon^{2}\right)$ an equation of the form $f+\varepsilon g$, where $g \in R$. We say that $g$ represents a tangent vector to a flat deformation of $D$ as a subscheme of $U$. To save space denote $\mathbf{C}[\varepsilon] /\left(\varepsilon^{2}\right)$ by $\mathrm{C}[\varepsilon]$.

(4.9) Proposition. Continue with the notation of the preceding definition. Assume $D$ is singular at a point $p$. Let $A, H, I$, and $J$ be the conductor, equiclassical, equisingular, and Jacobian ideals of $D$ at $p$, thought of as ideals in $R$. (By this we mean the ideal in $R$ obtained by contraction from the ideal in the local ring of $D$ at $p$.)

(1) If $\pi: X \rightarrow Y$ is formally locally trivial in a neighborhood of $y \times p$ then $g \in J$.

(2) If $\pi: X \rightarrow Y$ is equisingular or formally locally equisingular in a neighborhood of $y \times p$ then $g \in I$.

(3) Assume $Y$ is reduced. If $\pi: X \rightarrow Y$ is equiclassical in a neighborhood of $y \times p$ and $Y$ is regular at $y$ then $g \in H$. (Without the regularity assumption there are cases where $g \notin H$.)

(4) Assume $Y$ is reduced. If $\pi: X \rightarrow Y$ is equigeneric in a neighborhood of $y \times p$ and $Y$ is regular at $y$ then $g \in A$. (Without the regularity assumption there are cases where $g \notin A$.)

ProOF. In any case the complete local ring of $U$ at $p$ is $\mathbf{C}[[x, y]]$. The family

$$
\begin{aligned}
& X^{\prime} \subset U \times \operatorname{Spec} \mathbf{C}[\varepsilon] \\
& \downarrow \\
& \text { Spec } \mathbf{C}[\varepsilon]
\end{aligned}
$$

with local equation $f+\varepsilon g$ gives a family of algebroid plane curves

$$
\begin{aligned}
& X^{\prime \prime} \subset \operatorname{Spec} \mathbf{C}[[x, y]] \times \operatorname{Spec} \mathbf{C}[\varepsilon] \\
& \downarrow \\
& \operatorname{Spec} \mathbf{C}[\varepsilon]
\end{aligned}
$$


with equation $f+\varepsilon g$, where now by $f$ and $g$ we mean their images in $\mathbf{C}[[x, y]]$. Furthermore if the original family was formally locally trivial, equisingular, or formally locally equisingular in a neighborhood of $y \times p$ then the family $X^{\prime \prime} \rightarrow \operatorname{Spec} \mathbf{C}[\varepsilon]$ will have the same property. The family $X^{\prime \prime} \rightarrow \operatorname{Spec} \mathbf{C}[\varepsilon]$ gives a morphism $q: \operatorname{Spec} \mathbf{C}[\varepsilon] \rightarrow \operatorname{Spec} \mathbf{C}\left[\left[t_{1}, \ldots, t_{m}\right]\right]$, where Spec $\mathbf{C}\left[\left[t_{1}, \ldots, t_{m}\right]\right]$ is the base of the formal versal deformation space. The corresponding homomorphism $q^{\prime}: \mathbf{C}\left[\left[t_{1}, \ldots, t_{m}\right]\right]$ $\rightarrow \mathbf{C}[\varepsilon]$ may be defined as follows. Write $g$ as $h+\sum a_{i} g_{i}$ where $h \in J, a_{i} \in \mathbf{C}$, and the $g_{i}$ are the chosen basis for $\mathbf{C}[[x, y]] / J$, and set $t_{i} \rightarrow a_{i} \varepsilon$. Clearly the family $X^{\prime \prime} \rightarrow \operatorname{Spec} \mathbf{C}[\varepsilon]$ is formally locally trivial if and only if it is trivial. By (3.18) the family is trivial if and only if the homomorphism $q^{\prime}$ is the zero homomorphism, that is if and only if $g \in J$. Since the $J$ in $R$ comes by contraction from the $J$ in $\mathbf{C}[[x, y]]$ this completes $(1)$. With these observations part (2) follows directly from (3.25).

Parts (3) and (4) are straightforward consequences of (4.4). To see the necessity of the regularity assumption in part (4) consider the family over $\operatorname{Spec} \mathbf{C}\left[t^{2}, t^{3}\right]$ of curves in $\mathbf{C}^{2}$ whose equation is

$$
y^{2}-x^{3}+t^{2} x+2 \sqrt{3} t^{3} / 9 \quad\left(=y^{2}-(x+\sqrt{3} t / 3)^{2}(x-2 \sqrt{3} t / 3)\right) .
$$

This family is clearly equigeneric. The homomorphism $\mathbf{C}\left[t^{2}, t^{3}\right] \rightarrow \mathbf{C}[\varepsilon]$ given by $t^{2} \rightarrow 0, t^{3} \rightarrow \varepsilon$ gives a family over Spec $\mathbf{C}[\varepsilon]$ with equation $y^{2}-x^{3}+2 \sqrt{3} \varepsilon / 9$. But clearly $2 \sqrt{3} / 9 \notin A$. The necessity of the regularity assumption in part (3) follows from a similar argument applied to the family over the locus $E C$ in the deformation space of the ramphoid cusp that was discussed in $\S 1$.

(4.10) Proposition. Continue with the notation of (4.8) and (4.9). Assume $R=\mathbf{C}[[x, y]]$ or $\mathbf{C}[x, y]$. Assume $D$ has only one singular point.

(1) Every element of $J$ represents a tangent vector to a flat deformation of $D$ as a subscheme of $U$ formally locally trivial in a neighborhood of $y \times p$. Furthermore $Y$ may be taken to be regular at $y$, or even a nonsingular variety when $R=\mathbf{C}[x, y]$.

(2) Every element of $I$ represents a tangent vector to a flat deformation of $D$ as a subscheme of $U$ equisingular in a neighborhood of $y \times p$. Furthermore $Y$ may be taken to be regular at $y$, or even a nonsingular variety when $R=\mathbf{C}[x, y]$.

(3) If $Y$ is restricted to be regular at $y$, then for some singularities there are elements of $H$ which do not represent a tangent vector to a flat deformation of $D$ as a subscheme of $U$ equiclassical in a neighborhood of $y \times p$.

(4) If $Y$ is restricted to be reduced and regular at $y$, then for some singularities there are elements of $A$ which do not represent a tangent vector to a flat deformation of $D$ as subscheme of $U$ equigeneric in a neighborhood of $y \times p$.

ProOF. For (1) we will do the case $R=\mathbf{C}[x, y]$; the case $R=\mathbf{C}[[x, y]]$ is very similar. We may as well assume $p=(0,0)$. Let $g(x, y) \in J$, say

$$
g(x, y)=q_{1}(x, y) f(x, y)+q_{2}(x, y) \partial f(x, y) / \partial x+q_{3}(x, y) \partial f(x, y) / \partial y
$$

In $\mathbf{A}_{t}^{1} \times \mathbf{A}_{x, y}^{2}$ consider the locus

$$
\left\{0=F(t, x, y)=\left(1+t q_{1}(x, y)\right) f\left(x+t q_{2}(x, y), y+t q_{3}(x, y)\right)\right\}
$$

$F(t, x, y)$ is a polynomial. Think of this as a family of curves over $\mathbf{A}_{t}^{1}$. Writing out the Taylor expansion for $F$ in powers of $t$ one sees that the coefficient of $t$ is $g(x, y)$. 
When $t=0$ the fiber is $\{f=0\}=D$. The family is formally locally trivial in some Zariski open subset of $0 \times(0,0)$ because we are just changing coordinates.

Again for (2) we will do the case $R=\mathbf{C}[x, y]$. Let $g(x, y) \in I$. Write $g(x, y)=$ $h(x, y)+\sum a_{i} g_{i}(x, y), h \in J, a_{i} \in \mathbf{C},\left\{g_{i}\right\}$ the chosen basis for $\mathbf{C}[x, y] / J$ in the construction of the étale versal deformation space. Write

$$
h(x, y)=q_{1}(x, y) f(x, y)+q_{2}(x, y) \partial f(x, y) / \partial x+q_{3}(x, y) \partial f(x, y) / \partial y .
$$

As usual let $B$ be the base of the étale versal deformation of the singularity of $D$ at $p$. By (3.28) we may find a smooth curve $C$ through $(0, \ldots, 0) \in B$ lying in $E S$ with tangent line at $(0, \ldots, 0)$ equal to the line spanned by $(0, \ldots, 0)$ and $\left(a_{1}, \ldots, a_{m}\right)$. Let $t$ be a local parameter on $C$ at $(0, \ldots, 0)$. Over some Zariski open subset of $C$, call it $C^{\circ}$, this gives a family

$$
\begin{aligned}
& X \subset C^{\mathrm{o}} \times \mathbf{A}_{x, y}^{2} \\
& \downarrow \\
& C^{\mathrm{o}}
\end{aligned}
$$

with equation of the form $F(t, x, y)=f(x, y)+t \sum a_{i} g_{i}+t^{2} \ldots$. Furthermore this family is equisingular in a neighborhood of $(0, \ldots, 0) \times(0,0)$ (after possibly more étale base changes). The desired family then has equation

$$
\left(1+t q_{1}(x, y)\right) F\left(t, x+t q_{2}(x, y), y+t q_{3}(x, y)\right) .
$$

For (3) consider the ramphoid cusp $y^{2}+x^{5}$. The étale versal deformation space for this singularity was discussed in $\S 1$. The locus $E C$ is one dimensional with a unibranched singularity at $(0, \ldots, 0)$. Let

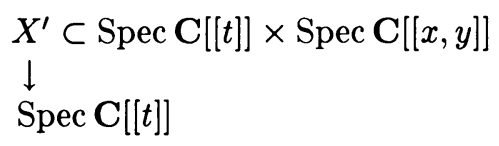

be an equiclassical family with equation $y^{2}+x^{5}+f_{1}(x, y) t+f_{2}(x, y) t^{2}+\cdots$. Let $\phi$ : Spec $\mathbf{C}[[t]] \rightarrow B^{\prime}$ be an induced map to the base of the formal versal deformation. The singularity of $E C^{\prime}$ at $(0, \ldots, 0)$ says that the differential of the map $\phi$ must vanish at $t=0$. In the proof of (4.9) we saw how to write down the map $\phi$ to first order. Doing this we find that the vanishing of the differential of $\phi$ implies that $f_{1} \in J$. But it is easy to see by direct computation that $H$ is strictly larger than $J$. The proof of (4) involves a similar argument applied to the singularity $y^{2}-x^{3}$ whose deformation space was also discussed in $\S 1$.

\section{(4.11) COROLlaRY. $J \subseteq I \subseteq H \subseteq A$.}

PROOF. $J \subseteq I$ was stated in (3.25). For $I \subseteq H$ notice that by (4.10) any element $g$ of $I$ comes from an equisingular deformation over a nonsingular base. Since an equisingular deformation is clearly equiclassical (4.9) says $g \in H . H \subseteq A$ follows easily from their definitions and the discussions at the beginning of $\S 4$.

(4.12) Definition. Let $D$ be a reduced projective curve in $\mathbf{P}^{2}$ and let $p_{1}, \ldots, p_{n}$ be the singular points of $D$. Let $S$ be a fixed subset of $p_{1}, \ldots, p_{n}$.

(1) We define a sheaf of ideals $J(D, S)$ on $\mathbf{P}^{2}$ as follows: for any open subset $U \subset \mathbf{P}^{2}, J(D, S)(U)=\left\{f \in \mathscr{O}(U): \forall p_{i} \in S\right.$ the image of $f$ in the complete local ring of $\mathbf{P}^{2}$ at $p_{i}$ lies in the Jacobian ideal of $D$ at $\left.p_{i}\right\} . J(D, S)$ will sometimes be denoted by $J(S)$. Similarly one can define $I(D, S), H(D, S)$, and $A(D, S)$. 
(2) Let

$$
\begin{gathered}
X \subset Y \times \mathbf{P}^{2} \\
\downarrow p \\
y \in Y
\end{gathered}
$$

be a flat family of reduced curves in $\mathbf{P}^{2}$ with $p^{-1}(y)$ equal to $D$ with its given embedding in $\mathbf{P}^{2}$.

(a) We say this family is trivial (equisingular) with respect to $S$ at $y$ in the Zariski (étale) topology if for each closed point $x \in p^{-1}(y) \cap S$ there exist Zariski (étale) open neighborhoods $U$ of $y$ in $Y$ and $V$ of $x$ in $p^{-1}(U)$ such that the family $V \rightarrow U$ is trivial (equisingular).

(b) We say this family is formally locally trivial (formally locally equisingular) with respect to $S$ at $y$ if for each positive integer $n$ the family $p_{n}: X \times_{Y} Y_{n} \rightarrow Y_{n}$ of (3.13)(4) is trivial (equisingular) with respect to $S$ at $y$ in the Zariski topology.

(c) Now assume that $Y$ is reduced at $y$. We say the family is equigeneric (equiclassical) with respect to $S$ at $y$ in the Zariski (étale) topology if given any closed point $x \in p^{-1}(y) \cap S$ and any open Zariski (étale) neighborhoods $U$ of $y$ in $Y$ and $V$ of $x$ in $p^{-1}(U)$ after possibly shrinking $U$ and $V$ to smaller neighborhoods $U^{\prime}$ and $V^{\prime}$ of $x$ and $y$ one may obtain a family $V^{\prime} \rightarrow U^{\prime}$ that is equigeneric (equiclassical).

The definition of formally locally trivial with respect to $S$ is essentially that of [Ta, p. 112].

Recall the following basic facts about curves of some fixed degree $d$ in $\mathbf{P}^{2}$. Fix homogeneous coordinates $x_{0}, x_{1}, x_{2}$ on $\mathbf{P}^{2}$. A curve $D$ of degree $d$ in $\mathbf{P}^{2}$ is then given by a homogeneous polynomial of degree $d, F=\sum a_{i, j, k} x_{0}^{i} x_{1}^{j} x_{2}^{k}$. All curves of degree $d$ in $\mathbf{P}^{2}$ are then parametrized by a projective space $\mathbf{P}^{N}$,

$$
N=\frac{1}{2}(d+2)(d+1)-1
$$

the correspondence being $D=\{F=0\} \mapsto\left[\cdots a_{i, j, k} \cdots\right]$. This $\mathbf{P}^{N}$ is the Hilbert scheme of curves of degree $d$ in $\mathbf{P}^{N}$ and as such the tangent space to the point of $\mathbf{P}^{N}$ corresponding to $D$ is naturally identified with $H^{0}\left(D, \mathscr{O}_{D}(d)\right)$. One may construct a universal curve over $\mathbf{P}^{N}$.

$$
\begin{gathered}
\mathbf{P}^{2} \times \mathbf{P}^{N} \supset \mathscr{C}=\left\{\sum a_{i, j, k} x_{0}^{i} x_{1}^{j} x_{2}^{k}=0\right\} \\
\downarrow \pi \\
\mathbf{P}^{N}
\end{gathered}
$$

Continue with the notation of (4.12). Let $d$ be the degree of $D$. From results in [Ta, pp. 109-113] and the universal mapping property of the Hilbert scheme one may deduce the following.

(4.13) FACT. Suppose that $H^{1}\left(D, \mathscr{O}_{D}(d) \otimes J(S)\right)=0$. Then there exists a subscheme $Z$ of $\mathbf{P}^{N}$ with the following properties.

(1) $Z$ contains the point $q$ corresponding to $D$.

(2) The restricted family $\pi: \mathscr{C} \rightarrow Z$ is formally locally trivial with respect to $S$ at $q$ in the Zariski topology.

(3) $Z$ is reduced and smooth at $q$ with tangent space naturally identified with $H^{0}\left(D, \mathscr{O}_{D}(d) \otimes J(S)\right)$.

(4) If $Z^{\prime}$ is any other subscheme of $\mathbf{P}^{N}$ satisfying (1) and (2) then, in some Zariski neighborhood of $q, Z^{\prime} \subset Z$. 
(4.14) Lemma [AC, Z1]. Let $U^{d, g}$ be the closure in $\mathbf{P}^{N}$ of the locus of points corresponding to reduced curves of degree $d$ and geometric genus $g$. Then every component of $U^{d, g}$ has dimension $3 d+g-1$.

(4.15) THEOREM. The tangent cone to $E G\left(E G^{\prime}\right)$ at $(0, \ldots, 0)$ is supported on a linear subspace of $B\left(B^{\prime}\right)$ which under the identification $B=\mathbf{C}[x, y] / J\left(B^{\prime}=\right.$ $\mathrm{C}[[x, y]] / J)$ is identified with $A / J$.

PROOF. The proofs for $E G$ and $E G^{\prime}$ are essentially the same. We do $E G$. Let $T$ be the support of the tangent cone to $E G$ at $(0, \ldots, 0)$. Lemma (4.4) says that $T \subset A / J$. Since $A / J$ is irreducible all we must do to prove equality is show that their dimensions are equal. Since $\operatorname{dim} T=\operatorname{dim} E G$ this is the same as showing $\operatorname{dim} E G=\operatorname{degree} J-\operatorname{degree} A$.

Notice that in the statement of the proposition we did not mention explicitly the curve and the singular point on it to which the deformation spaces we are considering correspond. This is because for a local question like this we may choose any curve and any singular point on it with an étale neighborhood isomorphic to an étale neighborhood of the one we are interested in. From (3.20) we see that we may choose a reduced projective curve $D$ of degree $d$ in $\mathbf{P}^{2}$ with only one singular point $p$, with $d$ sufficiently large so that $H^{1}\left(D, \mathscr{O}_{D}(d) \otimes J\right)=0$ and $\operatorname{dim} H^{0}\left(D, \mathscr{O}_{D}(d) \otimes J\right)=\frac{1}{2}(d+2)(d+1)$-degree $J-1$. Let $\mathbf{P}^{N}$ be the projective space parametrizing curves of degree $d$ in $\mathbf{P}^{2}$ and let $q$ be the point corresponding to $D$. By basic properties of the étale versal deformation we get étale neighborhoods $V$ of $(0, \ldots, 0)$ in $B$ and $U$ of $q$ in $\mathbf{P}^{N}$ and a morphism $\phi: U \rightarrow V$.

By (4.13) the tangent space to $\phi^{-1}(0, \ldots, 0)$ at $q$ is $H^{0}\left(D, \mathscr{O}_{D}(d) \otimes J\right)$. Putting this together with $\operatorname{dim} U-\frac{1}{2}(d+2)(d+1)-1, \operatorname{dim} V=$ degree $J$, one concludes that after possibly shrinking $U$ and $V$ we may assume that $\phi$ is surjective with smooth fibers of dimension $\frac{1}{2}(d+2)(d+1)-$ degree $J-1$. Notice that the geometric genus of $D$ is $g=\frac{1}{2}(d-1)(d-2)$-degree $A$. Clearly $\phi^{-1}(E G)=U^{d, g}$, so

$$
\operatorname{dim} E G=\operatorname{dim} U^{d, g}-\left(\frac{1}{2}(d+2)(d+1)-\operatorname{degree} J-1\right) .
$$

Using (4.14) this equals

$$
\begin{aligned}
3 d+ & -1-\frac{1}{2}(d+2)(d+1)+\text { degree } J+1 \\
& =3 d+\frac{1}{2}(d-1)(d-2)-\text { degree } A-\frac{1}{2}(d+2)(d+1)+\text { degree } J \\
& =\text { degree } J-\text { degree } A . \quad
\end{aligned}
$$

The fact that the support of the tangent cone to $E G$ at $(0, \ldots, 0)$ is irreducible puts some restrictions on what type of singularity $E G$ could have at $(0, \ldots, 0)$. Using the following results of Arbarello and Cornalba we can say even more.

(4.16) FACT. [AC, pp. 486-488]. Let $D$ be a reduced irreducible plane curve of degree $d$ in $\mathbf{P}^{2}$ of geometric genus $g$. Let $q$ be the point in $\mathbf{P}^{N}$ corresponding to $D$. (Use the usual metric topology on $\mathbf{C}^{n}$.)

(1) If all the singularities of $D$ have only smooth branches then there exists a neighborhood of $q$ in which $U^{d, g}$ is nonsingular.

(2) Assume $m(D)<3 d$. If any of the singularities of $D$ have any singular branches then $U^{d, g}$ is singular and unibranched at $q$. Furthermore a neighborhood of $q$ in $U^{d, g}$ is the one-one image of a smooth variety. 
(4.17) PROPOSITION. For a reduced plane curve singularity:

(1) $E G^{\prime}$ is irreducible. There is a Zariski neighborhood of $(0, \ldots, 0)$ in which $E G$ is irreducible.

(2) $E G\left(E G^{\prime}\right)$ is smooth at $(0, \ldots, 0)$ if and only if all the branches of the plane curve singularity are smooth.

(3) The normalization of $E G\left(E G^{\prime}\right)$ is smooth. There is a Zariski neighborhood of $(0, \ldots, 0)$ in which the normalization map is one-one.

PROOF. Using the ideas in the proof of (4.15) one sees that for an appropriate choice of $D$ and $p, U^{d, g}$ is-locally in the analytic topology-the product of $E G$ and a smooth variety. We may also choose $D$ to have sufficiently high degree $d$ so that $m(D)<3 d$. The results now follow immediately from (4.16).

(4.18) COROLLARY. In some Zariski open neighborhood of $(0, \ldots, 0) E C$ is a Zariski closed subset of EG; hence $E C^{\prime}$ is closed in $B^{\prime}$.

ProOF. Let $f: \widetilde{E G} \rightarrow E G$ be the normalization map. Since by (4.17) $\widetilde{E G}$ is nonsingular we may apply the discussion at the beginning of $\S 4$ to the family of curves over $\widetilde{E G}$ obtained via pull back from the étale versal family. In particular the paragraph before (4.3) says that $f^{-1}(E C)$ is a locally closed subset of $\widetilde{E G}$. But by $(4.16)(2)$ we may assume $f$ is one-one, hence a Zariski homeomorphism.

To end this section we present a result on tangent spaces and tangent cones that will be needed in the final section of this paper.

(4.19) Proposition. Let $D$ be a reduced curve of degree $d$ in $\mathbf{P}^{2}, S$ some subset of its singularities, and $q$ the point in $\mathbf{P}^{N}$ corresponding to $D$. Let $Z$ be a subscheme of $\mathbf{P}^{N}$ which contains $q$.

(1) If the restriction of the universal family $\pi: \mathscr{C} \rightarrow \mathbf{P}^{N}$ to $\pi: \pi^{-1}(Z) \rightarrow Z$ is trivial or formally locally trivial with respect to $S$ at $q$ in either the Zariski or étale topology, then the tangent space to $Z$ at $q$ is contained in $H^{0}\left(D, \mathscr{O}_{D}(d) \otimes J(S)\right)$. Also there is some such $Z$ whose tangent space at $q$ equals $H^{0}\left(D, \mathscr{O}_{D}(d) \otimes J(S)\right)$.

(2) Same as (1) except replace trivial with equisingular, formally locally trivial with formally locally equisingular, and $J(S)$ with $I(S)$.

(3) Assume that $Z$ is reduced. If the restriction of the universal family $\pi: \mathscr{C} \rightarrow$ $\mathbf{P}^{N}$ to $\pi: \pi^{-1}(Z) \rightarrow Z$ is equigeneric with respect to $S$ at $q$ in either the Zariski or étale topology, then the support of the tangent cone to $Z$ at $q$ is contained in $H^{0}\left(D, \mathscr{O}_{D}(d) \otimes A(S)\right)$.

PROOF. Recall one of the ways of seeing that the tangent space to $\mathbf{P}^{N}$ at $q$ is isomorphic to $H^{0}\left(D, \mathscr{O}_{D}(d)\right)$. The tangent space to $\mathbf{P}^{N}$ at $q$ is isomorphic to equivalence classes of families of plane curves of degree $d$ over Spec $\mathbf{C}[\varepsilon]$ with central fiber $D$. If $F$ is the homogeneous equation of $D$ such a family has an equation $F+\varepsilon G$ where $G$ is a homogeneous polynomial of degree $d$ or the zero polynomial. $F+\varepsilon G$ and $F+\varepsilon H$ are equivalent families if and only if $G$ and $H$ have the same image in $H^{0}\left(D, \mathscr{O}_{D}(d)\right)$.

Now suppose we have a $Z$ satisfying the hypothesis of (1). $F+\varepsilon G$ represents a tangent vector to $Z$ at $q \Rightarrow$ the family $F+\varepsilon G$ is trivial with respect to $S$ at $q \Leftrightarrow$ for all $p \in S$ the induced family of algebroid plane curves near $p$ is trivial $\Leftrightarrow$ for all $p \in S$ the image of $G$ in the complete local ring of $\mathbf{P}^{2}$ at $p$ is in the Jacobian ideal of $D$ at $p \Leftrightarrow G$ represents an element of $H^{0}\left(D, \mathscr{O}_{D}(d) \otimes J(S)\right)$. 
For the "also..." part of (1) let $G_{1}, \ldots, G_{m}$ be homogeneous polynomials of degree $d$ which give a basis for $H^{0}\left(D, \mathscr{O}_{D}(d) \otimes J(S)\right)$. The family over

$$
\operatorname{Spec} \mathbf{C}\left[\varepsilon_{1}, \ldots, \varepsilon_{m}\right] /\left(\varepsilon_{i} \varepsilon_{j}\right)
$$

with equation $F+\sum \varepsilon_{i} G_{i}$ can be seen to be trivial with respect to $S$ by the reasoning in the previous paragraph. It is clear that one may embed $\operatorname{Spec} \mathbf{C}\left[\varepsilon_{1}, \ldots, \varepsilon_{m}\right] /\left(\varepsilon_{i} \varepsilon_{j}\right)$ in $\mathbf{P}^{N}$ supported at $q$ to give the desired $Z$.

Part (2) proceeds as in (1) with the obvious minor changes.

For part (3) notice that $H^{0}\left(D, \mathscr{O}_{D}(d)\right)$ can also be thought of as the tangent cone to $\mathbf{P}^{N}$ at $q$. Consider families of plane curves of degree $d$ over Spec $\mathbf{C}[[t]]$ with central fiber $D$. Such a family has an equation of the form $F+t F_{1}+t^{2} F_{2}+\cdots$ where $F$ is a homogeneous equation for $D$ and the $F_{i}$ are either zero or homogeneous polynomials of degree $d$. If $i$ is the smallest integer for which $F_{i} \neq 0$ and $F_{i}$ is not a multiple of $F$ (this can be arranged by reparametrizing the family if necessary) then the image of $F_{i}$ in $H^{0}\left(D, \mathscr{O}_{D}(d)\right)$ spans a line through the origin which is the line in the tangent cone to $\mathbf{P}^{N}$ at $q$ given by this family. Given a $Z$ satisfying the hypothesis of (3) then all lines in the support of the tangent cone to $Z$ at $q$ come from such families that are equigeneric with respect to $S$. $F+t F_{1}+t^{2} F_{2}+\cdots$ is equigeneric with respect to $S$ if and only if for all $p \in S$ the induced family of algebroid plane curves near $p$ is equigeneric. By (4.4) this is the case only if for all $p \in S$ the image of the first nonzero $F_{i}$ in the complete local ring of $\mathbf{P}^{2}$ at $p$ lies in the conductor ideal of $D$ at $p$; this is true if and only if this first nonzero $F_{i}$ represents an element of $H^{0}\left(D, \mathscr{O}_{D}(d) \otimes A(s)\right)$.

5. Equiclassical deformations. In this section we study equiclassical families in greater detail.

(5.1) LEMMA. Consider a family of curves as at the beginning of $\S 4$. Define $M_{k}=\left\{y \in Y: m\left(\pi^{-1}(y)\right) \geq k\right\}$. See (3.12) for the definition of $m$. Then the codimension of $M_{k}$ in $Y$ is at most $k$.

PROOF. We continue with the notation of the discussion at the beginning of $\S 4$. The paragraph before (4.3) tells us that $M_{k}$ is a closed subset of $Y$. The family $\pi \circ h: X^{\prime} \rightarrow Y$ is a family of nonsingular curves. One may construct a family $\pi_{k}: X^{\prime(k)} \rightarrow Y$ where the fiber over $y \in Y$ is the $k$ th symmetric product of the curve $(\pi \circ h)^{-1}(y)$. We represent a point on $X^{\prime(k)}$ by $(D, y)$ where $D$ is an effective divisor on $(\pi \circ h)^{-1}(y)$. There also exist a family of curves $f: Z \rightarrow X^{\prime(k)}$ such that $f^{-1}(D, y)=(\pi \circ h)^{-1}(y)$ and a divisor $\Delta$ on $Z$ such that the restriction of $\Delta$ to $f^{-1}(D, y)$ is $D$. Recall the divisor $\mathscr{R}_{y}$ of $(4.2)$. Define $\Gamma=\left\{(D, y): \mathscr{R}_{y} \geq D\right\}$. Clearly $\pi_{k}(\Gamma)=M_{k}$.

Locally $\Gamma$ may be described as follows. Pick a point $\left(D_{0}, y_{0}\right)$. Let $z$ be a local coordinate on $(\pi \circ h)^{-1}\left(y_{0}\right)$ in a neighborhood of $D_{0}$ and let $x_{1}$ and $x_{2}$ be local coordinates on $\mathbf{P}^{2}$ in a neighborhood of $D_{0}$. Then, near $\left(D_{0}, y_{0}\right), \Gamma=\left\{(D, y): \partial x_{1} / \partial z\right.$ and $\partial x_{2} / \partial z$ both vanish on $\left.D\right\}$. Locally $\partial x_{i} / \partial z \in \mathscr{O}_{Z}$. Via the natural map $f_{*} \mathscr{O}_{Z} \rightarrow f_{*} \mathscr{O}_{\Delta}, \partial x_{i} / \partial z$ gives locally a section of $f_{*} \mathscr{O}_{\Delta}$; this section vanishes exactly on $\Gamma_{i}=\left\{(D, y): \partial x_{i} / \partial z\right.$ vanishes on $\left.D\right\}$. Since $f_{*} \mathscr{O}_{\Delta}$ is a vector bundle of rank $k$ the set $\Gamma_{i}$ if nonempty has codimension at most $k$ in $X^{\prime(k)}$, so $\Gamma=\Gamma_{1} \cap \Gamma_{2}$ if nonempty has codimension at most $2 k$ in $X^{\prime(k)}$. $\mathscr{R}_{y}$ is always a divisor on 
$\langle\pi \circ h)^{-1}(y)$. Thus the map $\pi_{k}: \Gamma \rightarrow Y$ has finite fibers, so the codimension of $M_{k}=\pi_{k}(\Gamma)$, if nonempty, in $Y$ is at most $k$.

(5.2) COROLlaRY. Let $\mathbf{P}^{N}$ be the projective space that parametrizes curves of degree $d$ in $\mathbf{P}^{2}$. Let $V^{d, g, c}$ be the locus of points of $\mathbf{P}^{N}$ corresponding to reduced irreducible curves of geometric genus $g$ and class $c$. Assume that $2 g-d-2<c$. Then if $V^{d, g, c}$ is nonempty then every irreducible component of $V^{d, g, c}$ has dimension at least $d+c-g+1$.

PrOOF. From the classical formulas $g=\frac{1}{2}(d-1)(d-2)-\delta, c=d(d-1)-2 \delta-m$, we see that $2 g-d-2<c$ is equivalent to $m<3 d$. This says that (4.16)(2) applies here. Applying an argument similar to the one in the proof of (4.18) one can show that $V^{d, g, c}$ is a locally closed subset of $\mathbf{P}^{N}$.

By definition each component of $V^{d, g, c}$ is a subset of a component of $V^{d, g}$. By (4.14) we know every component of $V^{d, g}$ has dimension $3 d+g-1$. We must calculate the codimension of $V^{d, g, c}$ in $V^{d, g}$. By (4.16) we know that the normalization of $V^{d, g}$ is nonsingular. This says that (5.1) holds on the pull back of the universal curve over $\mathbf{P}^{N}$ to the normalization of $V^{d, g}$. Since we have the classical formulas $g=\frac{1}{2}(d-1)(d-2)-\delta, c=d(d-1)-2 \delta-m$, simple algebra finishes the proof.

(5.3) LEMMA. Let $D$ be a reduced plane curve whose only singularity is at $(0,0)$. Then, for $D$, degree $H=\operatorname{dim}_{\mathbf{C}} \mathbf{C}[x, y] / H=\delta+m$.

PROOF. The first equality is an elementary fact about ideals, so all we have to prove is the second.

We may assume $D$ is a curve in $\mathbf{P}^{2}$ with only one singularity. Let $D^{\prime}$ be the normalization of $D$. Let $A^{\prime}$ and $R^{\prime}$ be the divisors on $D^{\prime}$ from the discussion at the beginning of $\S 4$. We have the following commutative diagram for $k \geq 1$.

$$
\begin{array}{ccc}
H^{0}\left(D, \mathscr{O}_{D}(k) \otimes A\right) & \hookrightarrow & H^{0}\left(D^{\prime}, \mathscr{O}_{D^{\prime}}(k)-A^{\prime}\right) \\
\cup & \cup & \cup \\
H^{0}\left(D, \mathscr{O}_{D}(k) \otimes H\right) & \hookrightarrow & H^{0}\left(D^{\prime}, \mathscr{O}_{D^{\prime}}(k)-A^{\prime}-R^{\prime}\right)
\end{array}
$$

From [ACGH, pp. 57-60] we see that the top inclusion is an isomorphism. Using this one easily shows that the bottom inclusion is also an isomorphism. One also sees from [ACGH, pp. 57-60] that degree $A^{\prime}=2 \delta$.

For $k$ sufficiently large

$$
\operatorname{dim} H^{0}\left(D, \mathscr{O}_{D}(k) \otimes H\right)=\frac{1}{2}(k+2)(k+1)-\frac{1}{2}(k-d+2)(k-d+1)-\operatorname{deg} H-1
$$

and by Riemann-Roch

$$
\operatorname{dim} H^{0}\left(D^{\prime}, \mathscr{O}_{D^{\prime}}(k)-A^{\prime}-R^{\prime}\right)=k d-2 \delta-m-g+1 .
$$

Equating these two expressions, putting in $g=\frac{1}{2}(d-1)(d-2)-\delta$, and simplifying completes the proof.

(5.5) THEOREM. The tangent cone to $E C\left(E C^{\prime}\right)$ at $(0, \ldots, 0)$ is supported on a linear subspace of the tangent space $T$ to $B\left(B^{\prime}\right)$ at $(0, \ldots, 0)$ which under the identification $T=\mathbf{C}[x, y] / J(\mathbf{C}[[x, y]] / J)$ is identified with $H / J$.

Proof. The proof is almost identical to the proof of (4.15) with $E C$ taking the place of $E G, H$ taking the place of $A$, and (5.2) taking the place of (4.14). Also use (5.3). 
(5.6) Proposition [W, p. 160]. For the singularity $y^{p}+x^{q}=0(p \leq q)$, the equisingular ideal $I$ is the ideal generated by $x^{q-1}, y^{p-1}$, and the monomials $x^{i} y^{j}$, where $p i+q j \geq p q$.

(5.7) LEMMA. For the singularity $y^{p}+x^{q}=0(p \leq q), p$ and $q$ relatively prime, the equiclassical ideal $H$ is the ideal generated by the monomials $x^{i} y^{j}$, where $p i+q j \geq p q-q$.

PROOF. Since $p$ and $q$ are relatively prime this singularity is unibranched. This curve is given parametrically by $x=t^{p}, y=t^{q}, t$ a coordinate on the normalization. The pull back of the Jacobian ideal to the normalization is the principal ideal $\left(t^{p q-q}\right)$. A monomial $x^{i} y^{j}$ pulled back to the normalization becomes $t^{p i} t^{q j}=t^{p i+q j}$, so $x^{i} y^{j} \in H$ if and only if $p i+q j \geq p q-q$.

(5.8) COROLlaRY. For the singularity $y^{p}+x^{q}=0(p \leq q), p$ and $q$ relatively prime, $I \varsubsetneqq H$ except when $p=2, q=3$. bra.

PROOF. This follows from (5.6), (5.7) and the elementary high school alge-

Let $f: X \rightarrow Y$ be a flat family of reduced plane curves with $Y$ nonsingular and of finite type over $\mathbf{C}$. Let $y \in Y$ be a closed point. Assume that the family $f: X \rightarrow Y$ is versal in the étale topology for $f^{-1}(y)$ at $y$. Also assume for simplicity that $f^{-1}(y)$ has only one singular point. Construct $\pi: \mathscr{C} \rightarrow B$, the étale versal deformation space for $f^{-1}(y)$, as at the beginning of $\S 3$. Then there exist étale open neighborhoods $V$ of $(0, \ldots, 0)$ in $B$ and $V^{\prime}$ of $y$ in $Y$ and a morphism $g: V^{\prime} \rightarrow V$ such that (3.3) is satisfied. By examining the differential of the map $g$ at $y$ and using (3.4) and the fact that the formal versal deformation is a universal first order deformation one may deduce that near $y g$ is surjective with smooth fibers of constant dimension. Of course the family $f: X \rightarrow Y$ is locally trivial along these fibers.

Near $y$ define $E C_{y}=\left\{z \in Y: \kappa\left(f^{-1}(z)\right)=\kappa\left(f^{-1}(y)\right) ; \delta\left(f^{-1}(y)\right)=\delta\left(f^{-1}(z)\right)\right\}$. Using what we have just said one sees that:

$$
\text { codimension of } E C_{y} \text { in } Y=\text { codimension of } E C \text { in } B \text {. }
$$

The same is true for any other subvariety defined by a reasonable geometric condition on the fibers, for instance $E S$ and $E G$.

(5.10) Proposition. For a singularity of a reduced plane curve, $I \varsubsetneqq H$ if and only if ES is a proper subvariety of EC. (By this we mean that ES is contained in EC and is not equal to any irreducible component of EC.)

Proof. It is clear that ES $\subset E C$. From (3.25), (3.28), and (5.5) we see that the if part of the proposition is true.

Let $D$ be a reduced plane curve whose only singularity is the one under consideration. Using $D$ construct the étale versal deformation $\pi: \mathscr{C} \rightarrow B$ for $D$; in $B$ we have $E S$ and $E C$. The assumption $I \varsubsetneqq H$ together with (3.25), (3.28), and (5.5) tells us that through $(0, \ldots, 0)$ there is a component of $E C$ of dimension strictly greater than the dimension of ES. From (5.3) and (5.5) we see that the codimension of the equiclassical locus in the base of the étale versal deformation is the same for equivalent singularities; (3.5) and (5.9) and the third sentence of this paragraph tell us that through any point of $E S$ near $(0, \ldots, 0)$ there is a component of $E C$ 
of dimension strictly greater than the dimension of $E S$. This says that $E S$ is a proper subvariety of $E C$.

(5.11) FACT. Let $D$ be a reduced plane algebroid curve and $p$ the closed point of $D$. Let $f: D^{\prime} \rightarrow D$ be the normalization map and $D_{1}^{\prime}, \ldots, D_{n}^{\prime}$ the irreducible components of $D^{\prime}$. Then

$$
\begin{aligned}
\delta(D) & =\sum_{i=1}^{n} \delta\left(f\left(D_{i}^{\prime}\right)\right)+\sum_{i>j}\left[\text { intersection multiplicity of } f\left(D_{i}^{\prime}\right) \text { and } f\left(D_{j}^{\prime}\right) \text { at } p\right] \\
m(D) & =\sum_{i=1}^{n} m\left(f\left(D_{i}^{\prime}\right)\right) .
\end{aligned}
$$

PROOF. The first equation is classical; cf. [Hi, p. 183]. The second equation is obvious.

(5.12) THEOREM. Up to analytic isomorphism the only singularities of reduced plane curves for which $H=I$ are the ordinary node (local analytic equation $x y=0$ ) and the ordinary cusp (local analytic equation $y^{2}-x^{3}=0$ ). Equivalently, for these two singularities $E S=E C$ and for all others $E S$ is a proper subvariety of $E C$.

ProOF. Proposition (5.10) says that the two statements in the theorem are equivalent. It is a simple matter to check by direct computation that for an ordinary node and an ordinary cusp $H=I$ and $E C=E S$. We want to show that these are the only such singularities.

Claim 1. A singularity other than an ordinary node for which $E S$ is not a proper subvariety of $E C$ must have only one analytic branch.

First we exhibit for any singularity with two or more analytic branches, other than an ordinary node, a deformation that is equiclassical but not equisingular. Since we are free to take étale neighborhoods we may assume that the equation for the singularity factors, say $f(x, y) g(x, y)=0$. Let $U$ be the étale open set of $\mathbf{A}^{2}$ on which this factoring takes place. Choose a vector $(a, b) \in \mathbf{C}^{2}$. Consider the family

$$
\begin{aligned}
X & \subset U_{x, y} \times \mathbf{A}_{s, t}^{2} \\
& \downarrow \\
\mathbf{A}_{s, t}^{2} &
\end{aligned}
$$

with equation $f(x+a s, y+b t) g(x, y)=0$. For a sufficiently general choice of $(a, b)$ this family will not be equisingular because the singularity will have broken up into more than one singularity. This family will be equiclassical near $(0,0)$ because of (5.11) and the fact that we have not changed the contribution to the class of each branch nor the intersection multiplicities between the branches.

Using what we have just shown and (3.5) we see that in the étale versal deformation space of any multibranched singularity other than an ordinary node in a neighborhood of $(0, \ldots, 0)$ every point of $E S$ is in the closure of $E C-E S$. This proves Claim 1.

Claim 2. For any unibranched singularity that is not equivalent to $y^{p}+x^{p+1}=0$ for some integer $p \geq 2 E S$ is a proper subvariety of a component of $E C$.

As in Claim 1 all we must do is show that any such singularity has deformations that are equiclassical but not equisingular. 
Let $D \subset \mathbf{A}_{x, y}^{2}$ be a reduced plane curve with a unibranched singularity at $(0,0)$. Let $f: D^{\prime} \rightarrow D$ be the normalization map. Near $f^{-1}(0,0)$ the map $f$ may be expressed as the composition of an embedding $\phi: D^{\prime} \rightarrow \mathbf{A}^{m}$ and a projection $\pi: \mathbf{A}^{m} \rightarrow \mathbf{A}^{2}$. Further we may assume that $\phi\left(f^{-1}(0,0)\right)=(0, \ldots, 0)$ and that if $t$ is a local coordinate on $D^{\prime}$ at $f^{-1}(0,0) \phi$ is given by $\phi(t)=\left(t, t^{2}, \ldots, t^{m}\right)$. Let $z_{1}, \ldots, z_{m}$ be coordinates on $\mathbf{A}^{m}$ and let $p$ be the multiplicity of $(0,0)$ in $D$. Set $P_{k}=\left\{z_{m}=z_{m-1}=\cdots=z_{m-k+1}=0\right\} . P_{k}$ is the unique $m-k$ plane in $\mathbf{A}^{m}$ with contact of order $m-k+1$ with $\phi\left(D^{\prime}\right)$ at $(0, \ldots, 0)$. Let $P$ be the $m-3$ plane from which we project to obtain the map $\pi$ (we may assume $(0, \ldots, 0) \notin P$ ); let $P^{\prime}$ be any other $m-3$ plane and $\pi^{\prime}$ the resulting projection. Assume that $P^{\prime}$ does not contain $(0, \ldots, 0)$ and that $\pi^{\prime}\left(D^{\prime}\right)$ is unibranched at $\pi^{\prime}\left(f^{-1}(0,0)\right)$. The condition on $P^{\prime}$ that makes $\pi^{\prime}\left(D^{\prime}\right)$ have multiplicity $p$ at $\pi^{\prime}\left(f^{-1}(0,0)\right)$ is that $\pi^{\prime}$ collapse $P_{m-p+1}$ to a point. That is that $\operatorname{dim}\left(P^{\prime} \cap P_{m-p+1}\right)=p-2$. Among all $P^{\prime}$ that make $\pi^{\prime}\left(D^{\prime}\right)$ have multiplicity $p$ at $\pi^{\prime}\left(f^{-1}(0,0)\right)$ a Zariski open set will correspond to $P^{\prime}$ that meet all the $P_{k}$ as transversally as possible subject to the conditions $(0, \ldots, 0) \notin P^{\prime}, \operatorname{dim}\left(P^{\prime} \cap P_{m-p+1}\right)=p-2$. In particular, for a generic such $P^{\prime}$ we have

$$
\operatorname{dim}\left(P^{\prime} \cap P_{m-p}\right)=p-2, \quad \operatorname{dim}\left(P^{\prime} \cap P_{m-p-1}\right)=p-2 .
$$

$P^{\prime}$ is the common zeros of three linear equations. From the preceding conditions we see that for a generic $P^{\prime}$ that makes $\pi^{\prime}\left(D^{\prime}\right)$ have multiplicity $p$ at $\pi^{\prime}\left(f^{-1}(0,0)\right)$ the three equations for $P^{\prime}$ may be taken to be in the following form:

$F_{1}$ has nonzero constant term.

$F_{2}$ has no constant term and involves only $z_{p}, z_{p+1}, \ldots, z_{m}$ with the coefficient of $z_{p}$ nonzero.

$F_{3}$ has no constant term and involves only $z_{p+1}, \ldots, z_{m}$ with the coefficient of $z_{p+1}$ nonzero.

Therefore the parametric representation of $\pi^{\prime}\left(D^{\prime}\right)$ will be of the form

$$
\left(a_{1} t^{p}+a_{2} t^{p+1}+\cdots, b_{1} t^{p+1}+b_{2} t^{p+2}+\cdots\right)
$$

with $a_{1} \neq 0, b_{1} \neq 0$. It is easy to check that such a singularity is unibranched of multiplicity $p$ and resolves after a single blowup. Thus it is equivalent to $y^{p}+$ $x^{p+1}=0$. Consequently among all possible $P^{\prime}$ that give a unibranched singularity of multiplicity $p$ those that give a singularity equivalent to $y^{p}+x^{p+1}=0$ are open and dense.

If we move $P$ in a way that continues to give a unibranched singularity of multiplicity $p$ but is otherwise as general as possible the family of projections gives a family of plane curves with a special fiber equal to $D$ but each general fiber will have a singularity equivalent to $y^{p}+x^{p+1}=0$ and possibly other singularities. By construction this family has a simultaneous normalization; thus by [T1, 1.3.2] the family is equigeneric. Since $\kappa=2 \delta+m$ and $\delta$ is constant and every fiber has a unibranched singularity of multiplicity $p$ we see that a general fiber has $\kappa$ at least as large as the $\kappa$ for $D$. In $\S 4$ we saw that in any equigeneric family $\kappa$ is upper semicontinuous, so in the present family $\kappa$ must be constant and the family is equiclassical. By assumption the singularity of $D$ is not equivalent to $y^{p}+x^{p+1}=0$ so the family is not equisingular.

Claim 3. If $(D, p)$ and $(E, q)$ are equivalent unibranched singularities of reduced plane curves, then in the analytic topology there exists a flat equisingular family 
r: $X \rightarrow Y$ with $Y$ nonsingular and connected and two points $y_{1}, y_{2} \in Y$ such that $\pi^{-1}\left(y_{1}\right)$ is analytically isomorphic to $(D, p)$ and $\pi^{-1}\left(y_{2}\right)$ is analytically isomorphic to $(E, q)$.

This follows easily from the theory of Puiseaux expansions (cf. [T2, pp. 621622] for information on Puiseaux expansions). Equivalent singularities will have the same Puiseaux exponents; if the singularities are not analytically isomorphic the coefficients will be different. The desired family can be constructed by holomorphically varying the Puiseaux coefficients for one singularity into those for the other. (This proof was suggested by the referee and Bernard Teissier.)

Claim 4. If $(D, p)$ and $(E, q)$ are equivalent unibranched singularities of reduced plane curves and for $(D, p) E S$ is a proper subvariety of $E C$ then for $(E, q) E S$ is a proper subvariety of $E C$.

We already know that $E S$ and $E C$ are locally closed algebraic subsets of $B$ so we may work in the analytic topology.

From Claim 3 we know that there exists a flat equisingular family $\pi: X \rightarrow Y$ with $Y$ nonsingular and connected and two points $y_{1}, y_{2} \in Y$ such that $\pi^{-1}\left(y_{1}\right)$ is analytically isomorphic to $(D, p)$ and $\pi^{-1}\left(y_{2}\right)$ is analytically isomorphic to $(E, q)$. Let $\gamma:[0,1] \rightarrow Y$ be a continuous map with $\gamma(0)=y_{1}, \gamma(1)=y_{2}$. Call $X_{t}$ the fiber over $\gamma(t)$. For each $t \in[0,1]$ there is an open subset $U_{t} \subset Y$ such that the family $\pi: \pi^{-1}\left(U_{t}\right) \rightarrow U_{t}$ is pulled back from the versal deformation space for $X_{t}$. By compactness we may cover $\gamma([0,1])$ with a finite number of these $U_{t}$, call them $U_{t_{i}}$. Since the $U_{t_{i}}$ overlap we see that the fact that $E S$ is a proper subvariety of $E C$ for $(D, p)$ forces this to be true all along $\gamma([0,1])$ including at $(E, q)$.

Claim 5. For the singularity $y^{p}+x^{p+1}=0 E S$ is a proper subvariety of $E C$.

This is a direct consequence of (5.8) and (5.10).

These five claims prove the theorem.

(5.13) COROLLARY. For a reduced plane curve singularity a general point of $E C$ corresponds to a curve all of whose singularities are either ordinary nodes or ordinary cusps.

ProOF. This is an immediate consequence of (3.5), (3.6), and (5.12).

Corollary (5.13) implies (1.1). To prove (1.2) we need a few more results.

(5.14) Lemma. Let $D$ be a reduced and irreducible curve in $\mathbf{P}^{2}$ of degree $d$, geometric genus $g$ and class $c$. Let $H$ be its equiclassical ideal. Assume that $c \geq 2 g-1$. Then $H$ imposes independent conditions on curves of degree $d-1$ in $\mathbf{P}^{2}$.

ProOF. Using the formulas $g=\frac{1}{2}(d-1)(d-2)-\delta$ and $c=d(d-1)-2 \delta-m$ calculate that $c \geq 2 g-1$ says $m \leq 2 d-1$. Let $D^{\prime}$ be the normalization of $D$. From (5.4) and the discussion surrounding it we see that we have an isomorphism $H^{0}\left(D, \mathscr{O}_{D}(d-1) \otimes H\right) \cong H^{0}\left(D^{\prime}, \mathscr{O}_{D^{\prime}}(d-1)-A^{\prime}-R^{\prime}\right)$. The inequality $m \leq 2 d-1$ tells us that $\mathscr{O}_{D^{\prime}}(d-1)-A^{\prime}-R^{\prime}$ is nonspecial for degree reasons, so by RiemannRoch

$\operatorname{dim} H^{0}\left(D^{\prime}, \mathscr{O}_{D^{\prime}}(d-1)-A^{\prime}-R^{\prime}\right)=d(d-1)-2 \delta-m-\left(\frac{1}{2}(d-1)(d-2)-\delta\right)+1$.

The natural restriction map $H^{0}\left(\mathbf{P}^{2}, \mathscr{O}_{\mathbf{P}^{2}}(d-1) \otimes H\right) \rightarrow H^{0}\left(D, \mathscr{O}_{D}(d-1) \otimes H\right)$ is clearly injective. It is also surjective since $H^{0}\left(\mathbf{P}^{2}, \mathscr{O}_{\mathbf{P}^{2}}(d-1)\right) \rightarrow H^{0}\left(D, \mathscr{O}_{D}(d-1)\right)$ 
is surjective and $H$ is defined as an ideal in the local rings of the singular points of $D$ and becomes an ideal on $\mathbf{P}^{2}$ by contraction of ideals. This gives

$$
\begin{aligned}
\operatorname{dim} H^{0}\left(\mathbf{P}^{2}, \mathscr{O}_{\mathbf{P}^{2}}(d-1) \otimes H\right) & =\operatorname{dim} H^{0}\left(D, \mathscr{O}_{D}(d-1) \otimes H\right) \\
& =d(d-1)-2 \delta-m-\frac{1}{2}(d-1)(d-2)+\delta+1 .
\end{aligned}
$$

Using (5.3) this simplifies to

$$
\operatorname{dim} H^{0}\left(\mathbf{P}^{2}, \mathscr{O}_{\mathbf{P}^{2}}(d-1)\right)-\operatorname{deg} H .
$$

(5.15) THEOREM $[\mathbf{Z 1}$, pp. 213-215]. Let

$$
\begin{aligned}
& X \subset Y \times \mathbf{P}^{2} \\
& \downarrow \pi \\
& Y
\end{aligned}
$$

be a flat family of reduced curves in $\mathbf{P}^{2}$. Then there exists a Zariski open subset $U$ of $Y$ such that for each equivalence class of singularities all fibers over closed points of $U$ have the same number of singularities of that equivalence type. In fact these singularities are all equisingular specializations of singularities on the generic fiber of the family. By (3.22) we see that the family $\pi^{-1}(U) \rightarrow U$ is locally equisingular in the étale topology.

(5.16) FACT (MONOTONICITY OF HILBERT FUnCTIONS). Let $Z$ be a subscheme of $\mathbf{P}^{2}$ of dimension 0 and degree $n$. Let $m$ be a positive integer less than $n$. If $Z$ imposes at least $m$ conditions on curves of degree $d$ in $\mathbf{P}^{2}$, then $Z$ imposes at least $m+1$ conditions on curves of degree $d+1$ in $\mathbf{P}^{2}$.

Proof. Well known, cf. [DGM].

(5.17) ProOF OF (1.2). Let $W$ be the component of $Z$ on which $D$ lies. We may assume that $D$ is a nonsingular point of $W$. Let $S$ be the set of all singular points of $D$. By (4.19) and (5.15) we may assume that the tangent space to $W$ at $D$ is contained in $H^{0}\left(D, \mathscr{O}_{D}(d) \otimes I(S)\right)$. By (5.14) and (5.16)

$$
\begin{aligned}
\operatorname{dim} W & \leq \operatorname{dim} H^{0}\left(D, \mathscr{O}_{D}(d) \otimes I(S)\right) \\
& \leq \frac{1}{2}(d+2)(d+1)-1-\operatorname{deg} H(S)-\min \{1,(\operatorname{deg} I(S)-\operatorname{deg} H(S))\} .
\end{aligned}
$$

Using (5.3) and the formulas $g=\frac{1}{2}(d-1)(d-2)-\delta, c=d(d-1)-2 \delta-m$ this expression simplifies to

$$
d+c-g+1-\min \{1,(\operatorname{deg} I(S)-\operatorname{deg} H(S))\} .
$$

From (5.12) we see that if any of the singularities of $D$ are not ordinary nodes or ordinary cusps then $\operatorname{dim} W<d+c-g+1$. This contradicts (5.2).

6. Bounds for codimensions. In this section we study the relative position of $I$ between $J$ and $A$. In particular we give ways of estimating $\operatorname{dim}_{\mathbf{C}}(A / I)$ and give lists of singularities for which the number is 0,1 , or 2 . This allows us to prove statement (1.4) of the introduction.

From the previous section we know $J \subset I \subset A, \operatorname{dim} E S=\operatorname{dim}_{\mathbf{C}}(I / J)$, and $\operatorname{dim} E G=\operatorname{dim}_{\mathbf{C}}(A / J)$. This implies the following fact:

$$
\operatorname{dim}_{\mathbf{C}}(A / I)=\text { the codimension of } E S \text { in } E G \text {. }
$$


Of course the same is true for $E S^{\prime}$ and $E G^{\prime}$. One way to estimate the codimension of $E S$ in $E G$ is to look for sequences of subschemes

$$
E S=X_{0} \varsubsetneqq X_{1} \varsubsetneqq \cdots \varsubsetneqq X_{k-1} \varsubsetneqq X_{k}=E G
$$

in which we can estimate the codimension of $X_{i}$ in $X_{i+1}$. Such subschemes correspond to deformations that are equigeneric but not equisingular.

Let us fix some notation to be used until (6.9). $D=\{f(x, y)=0\}$ will be a reduced curve in $\mathbf{A}_{x, y}^{2}$ whose only singularity is at $(0,0) . A, I$, and $J$ will stand for the conductor, equisingular, and Jacobian ideals of $D$ at $(0,0)$. When other plane curves arise we will distinguish their ideals with subscripts, $A_{h}, I_{g}$, etc.

(6.3) LEMMA. If $D$ has $n$ analytic branches at $(0,0), n \geq 2$, then $\operatorname{dim}_{\mathbf{C}}(A / I)$ $\geq n-2$ with equality only if all the branches are smooth with distinct tangents.

ProOF. Since we are allowed to take étale neighborhoods we may assume that $f$ factors $f=f_{1} \cdots f_{n}$. In fact after étale base change we may consistently number the branches $1, \ldots, n$ over all of $E S$. If there are any branches tangent to each other assume that two of them correspond to $n-1$ and $n$.

Given a singularity with $n$ branches at the origin with equation $g_{1}(x, y) \cdots$ $g_{n}(x, y)=0$ one may construct the following family

$$
\begin{aligned}
& X \subset \mathbf{A}_{t_{1}, \ldots, t_{i}}^{i} \times \mathbf{A}_{x, y}^{2} \\
& \quad \mathbf{A}_{t_{1}, \ldots, t_{i}}^{i}
\end{aligned}
$$

which we call a family of type $i, 1 \leq i \leq n-2$. Choose $i$ general nonzero vectors in $\mathbf{C}^{2},\left(a_{1}, b_{1}\right), \ldots,\left(a_{n}, b_{n}\right)$. The equation for $X$ is

$$
g_{1}\left(x+a_{1} t_{1}, y+b_{1} t_{1}\right) \cdots g_{i}\left(x+a_{i} t_{i}, y+b_{i} t_{i}\right) g_{i+1}(x, y) \cdots g_{n}(x, y)=0 .
$$

We now define a sequence of subschemes as in (6.2). $X_{0}=E S, X_{n-1}=E G$. For $1 \leq i \leq n-2, X_{i}=$ the closure in $B$ of $X_{i}^{0}=\{q \in B$ : for each equivalence class of singularities $\pi^{-1}(q)$ has the same number of singularities of that equivalence type as a general fiber of a family of type $i$ with central fiber $D$ with branches numbered as we have numbered those of $D\} . X_{i}^{0}$ is contained in $E G$ because of (5.11) and the fact that we have not changed the contribution to the conductor of each branch, nor the total intersection multiplicities between the branches. By (5.15) we know that $X_{i}^{0}$ contains a Zariski open dense subset of $X_{i}$. Since (3.5) says the étale versal deformation remains versal in an open set around $(0, \ldots, 0)$ we see that for $i<n-2 X_{i}$ has codimension at least 1 in $X_{i+1}$. If any of the branches of $D$ are singular or tangent to each other a general point in $X_{n-2}$ will have singularities other than nodes. Since (1.3) and the argument in the proof of (4.15) allow us to conclude that a general point in $E G$ has only nodes as singularities, if any branches of $D$ are singular or tangent to each other $X_{n-2}$ has codimension at least 1 in $X_{n-1}=E G$.

(6.4) LEMMA. Suppose that at $(0,0) D$ has exactly two analytic branches, both smooth, meeting with contact of order $n$. Then $\operatorname{dim}_{\mathbf{C}}(A / I)=n-1$.

ProOF. We may assume $f(x, y)=y^{2}-x^{2 n}$. Notice that

$$
\operatorname{dim}_{\mathbf{C}}(A / I)=\text { degree } I-\operatorname{degree} A \text {. }
$$


Clearly degree $A=n-1$. From (5.6) one may calculate that $I=J$ and has degree $2 n-2$.

(6.5) It is also useful to see a sequence of subschemes as in (6.2) for this singularity. Take $X_{0}=E S=(0, \ldots, 0), X_{n-1}=E G$, and for $1 \leq i \leq n-2 X_{i}=$ the closure in $B$ of $X_{i}^{0}=\left\{q \in B\right.$ : the singularities of $\pi^{-1}(q)$ are one singularity consisting of two smooth branches meeting with contact of order $n-i$ and $i$ nodes $\}$.

(6.6) LEMMA. Assume $D$ has only one analytic branch at $(0,0)$ and $D$ has multiplicity $m$ at $(0,0)$. Blow up $(0,0)$.

(1) If $D^{\prime}$ (the proper transform of $\left.D\right)$ is smooth then $\operatorname{dim}_{\mathbf{C}}(A / I) \geq 2 m-3$.

(2) If $D^{\prime}$ is singular then $\operatorname{dim}_{\mathbf{C}}(A / I) \geq m$.

PROOF. (1) $D^{\prime}$ has contact of order $m$ with the exceptional divisor $E$. One may deform $D^{\prime}$ to a curve $D_{2}^{\prime}$ meeting $E$ once with contact of order $m-1$ and once transversely, then to $D_{3}^{\prime}$ meeting $E$ once with contact of order $m-2$ and twice transversely. Continue in this way until $D_{m}^{\prime}$ meets $E$ transversely in $m$ points. Let $D_{i}$ be the image of $D_{i}^{\prime}$ in $\mathbf{A}^{2}$. $D_{i}$ will have one singular point with $i$ branches; for $i<m$ one branch will have a cusp of some sort and the others will be smooth, and when $i=m$ all branches will be smooth. $D_{i}$ is an equigeneric deformation of $D$ because on the blowup we have kept intersection numbers with the exceptional divisor constant.

We now define a sequence of subschemes $E S=X_{1} \varsubsetneqq X_{2} \varsubsetneqq \cdots \varsubsetneqq X_{2 m-3} \varsubsetneqq$ $X_{2 m-2}=E G$. For $2 \leq i \leq m X_{i}$ is the closure in $B$ of all points of $B$ representing curves whose singularity is equivalent to the singularity of $D_{i}$. For $m+1 \leq i \leq$ $2 m-2 X_{i}$ is the closure in $B$ of all points of $B$ corresponding to curves whose singularities are one $m-(i-m)$ fold point with smooth branches with distinct tangent directions and $(i-m) m-\frac{1}{2}(i-m+1)(i-m)$ ordinary nodes. Argue as in the proof of (6.3) to complete the proof.

(2) $D^{\prime}$ may be translated slightly to get a curve $D_{2}^{\prime}$ meeting the exceptional divisor $m$ times transversely and having the same singularity as $D^{\prime}$. Let $D_{2}$ be the image of $D_{2}^{\prime}$ in $\mathbf{A}^{2}$. The singularities of $D_{2}$ will be a cusp like the one of $D^{\prime}$ and an $m$-fold point with $m$ smooth branches meeting transversely. $D_{2}$ is an equigeneric deformation of $D$ because in the blowup we have not changed the singularity of the proper transform nor the intersection number with the exceptional divisor.

We now define our sequence $E S=X_{1} \varsubsetneqq X_{2} \varsubsetneqq \cdots \varsubsetneqq X_{m} \varsubsetneqq X_{m+1}=E G$. For $2 \leq i \leq m, X_{i}$ is the closure in $B$ of all points of $B$ corresponding to curves whose singularities consist of a cusp equivalent to the cusp of $D_{2}$, an $m-(i-2)$ fold point with smooth branches with distinct tangent directions and $(i-2) m-\frac{1}{2}(i-2)(i-1)$ ordinary nodes. Argue as in the proof of (6.3) to complete the proof.

(6.7) REMARKS. The technique of blowing up a singularity then moving the proper transform can be found in [AO].

In practice in $(6.6)(2)$ if one knows something about the cusp of $D^{\prime}$ one can do better than the bound of $m$. Between $X_{m}$ and $X_{m+1}$ one can put subschemes corresponding to deformations of the cusp of $D^{\prime}$ that are equigeneric but not equisingular.

For complicated singularities one can often combine the techniques used in (6.3), (6.5), and (6.6) to give better bounds on $\operatorname{dim}_{\mathbf{C}}(A / I)$ than any one of them alone gives. 
Using what we have just proven in this section one can compile the following list of all the analytic isomorphism classes of singularities with $\operatorname{dim}_{\mathbf{C}}(A / I)=0,1$, or 2 .

(6.8) $0 \quad$ smooth, (simple) node $x y=0$.

1 (simple) cusp $y^{2}+x^{3}$, tacnode $y^{2}+x^{4}$, (ordinary) triple point $x^{3}+y^{3}$.

2 (ordinary) quadruple point $y(x-y)(x-2 y)(x-\alpha y), \alpha \in \mathbf{C}-$ $\{1,2\}$ (we have a one parameter family of analytic isomorphism classes of these), oscnode $y^{2}+x^{6}$, ramphoid cusp $y^{2}+x^{5}$, cusp with extra smooth branch $x\left(y^{2}+x^{3}\right)$, tacnode with extra smooth branch $x\left(y^{2}+x^{4}\right)$.

(6.9) LEMMA. Let $D$ be a reduced irreducible curve of degree $d$ in $\mathbf{P}^{2}$ and let $S$ be any subset (not necessarily proper) of its singularities. Then the ideal sheaf $A(S)$ imposes independent conditions on curves of degree $d-3$ in $\mathbf{P}^{2}$.

PROOF. This is a well-known classical result, cf. [ACGH, pp. 57-60].

(6.10) PROOF OF (1.4). We may assume that $D$ is a nonsingular point of $W$. Let $S$ be all singular points of $D$. By (4.19) and (5.15) we may assume that the tangent space to $W$ at $D$ is contained in $H^{0}\left(D, \mathscr{O}_{D}(d) \otimes I(S)\right)$. By $(6.9)$ and (5.16) we have

$$
\begin{aligned}
\operatorname{dim} & H^{0}\left(D, \mathscr{O}_{D}(d) \otimes I(S)\right) \\
& \leq \frac{1}{2}(d+2)(d+1)-1-\operatorname{degree} A(S)-\min \{3,(\text { degree } I(S)-\text { degree } A(S))\} \\
& =\operatorname{dim} V-\min \{3,(\text { degree } I(S)-\text { degree } A(S))\} .
\end{aligned}
$$

A look at the list (6.8) finishes the proof.

\section{REFERENCES}

[AO] Norbert A'Campo, Le groupe de monodromie du deploiement des singularites isolees de courbes planes. I, Math. Ann. 213 (1975), 1-32.

[AC] E. Arbarello and M. Cornalba, A few remarks about the variety of irreducible plane curves of given degree and genus, Ann. Sci. École Norm. Sup. (4) 16 (1983), 467-488.

[ACGH] E. Arbarello, M. Cornalba, P. A. Griffiths, and J. Harris, Geometry of algebraic curves, vol. I, Springer-Verlag, Berlin and New York, 1985.

[A1] M. Artin, Versal deformations and algebraic stacks, Invent. Math. 27 (1974), 165-189.

[A2] _ Deformations of singularities, Tata Institute of Fundamental Research, Bombay, 1976.

[DGM] E. Davis, A. Geramita, and P. Maroscia, Perfect homogeneous ideals: Dubreil's theorems revisited, Bull. Sci. Math. (2) 108, (1984), 143-185.

[H] J. Harris, On the Severi problem, Invent. Math. 84 (1986), 445-461.

[Ha] R. Hartshorne, Algebraic geometry, Springer-Verlag, Berlin and New York, 1977.

[Hi] H. Hironaka, Arithmetic genera and effective genera of algebraic curves, Mem. Coll. Sci. Univ. Kyoto Sect. A30 (1956), 177-195.

[KS] A. Kas and M. Schlessinger, On the versal deformation of a complex space with an isolated singularity, Math. Ann. 196 (1972), 23-29.

[P] R. Piene, Polar classes of singular varieties, Ann. Sci. École Norm. Sup (4) 11 (1978), 247-276.

[S1] M. Schlessinger, Functors of Artin rings, Trans. Amer. Math. Soc. 130 (1968), 208-222.

[S2] _ _ Infinitesimal deformations of singularities, Thesis, Harvard Univ., 1964.

[Ta] A. Tannenbaum, Families of algebraic curves with nodes, Compositio Math. 41 (1980), 107126. 
[T1] B. Teissier, Resolution simultanee. I, II. Seminaire sur les Singularites des Surfaces Palaiseau, France 1976-1977, (M. Demazure et. al., eds.), Lecture Notes in Math., vol. 777, SpringerVerlag, Berlin and New York, 1980.

[T2] _ , The hunting of invariants in the geometry of discriminants, Real and Complex Singularities, (P. Holm., ed.), Sijthoff \& Noordhoff, 1977.

[W] J. Wahl, Equisingular deformations of plane algebroid curves, Trans. Amer. Math. Soc. 193 (1974), 143-170.

[Z1] O. Zariski, Dimension-theoretic characterization of maximal irreducible algebraic systems of plane nodal curves of a given order $n$ and with a given number $d$ of nodes, Amer. J. Math. 104 (1982), 209-226.

$[\mathbf{Z 2}] \_$, Equivalent singularities of plane algebroid curves, Amer. J. Math. 87 (1965), 507-536.

Department of Mathematics, Brandeis University, Waltham, MassachuSETTS 02254

Department of Mathematics, Brown University, Providence, Rhode Island 02912

Current address (Steven Diaz): Department of Mathematics, University of Pennsylvania, Philadelphia, Pennsylvania 19104-6395

Current address (Joe Harris): Department of Mathematics, Harvard University, Cambridge, Massachusetts 02138 